\title{
VOLUME PRESERVING MEAN CURVATURE FLOW FOR STAR-SHAPED SETS
}

\author{
INWON KIM AND DOHYUN KWON
}

\begin{abstract}
We study the evolution of star-shaped sets in volume preserving mean curvature flow. Constructed by approximate minimizing movements, our solutions preserve a strong version of starshapedness. We also show that the solutions converges to a ball as time goes to infinity. For asymptotic behavior of the solutions we use the gradient flow structure of the problem, whereas a modified notion of viscosity solutions is introduced to study the geometric properties of the flow by moving planes method.
\end{abstract}

\section{INTRODUCTION}

Let $\Omega_{0}$ be a open and bounded domain in $\mathbb{R}^{n}$ with unit volume, and consider the evolution of sets $\left(\Omega_{t}\right)_{t \geq 0}$ moving with the normal velocity $V$ given by

$$
V=-H+\lambda(t) \text { on } \Gamma_{t}:=\partial \Omega_{t}, \quad\left|\Omega_{t}\right|=\left|\Omega_{0}\right| .
$$

In smooth setting, $H$ is mean curvature on $\partial \Omega_{t}$ where $H$ is set to be positive if the domain is convex at the point, and $\lambda: \mathbb{R}_{0}^{+} \rightarrow \mathbb{R}$ satisfies $\int_{\Gamma_{t}} V d S=0$ so that the evolution satisfies $\left|\Omega_{t}\right|=\left|\Omega_{0}\right|$, i.e.

$$
\lambda(t)=\frac{1}{\operatorname{Per}\left(\Omega_{t}\right)} \int_{\partial \Omega_{t}} H d \sigma=f_{\partial \Omega_{t}} H d \sigma .
$$

There are two main difficulties to study the global behavior of the flow (1.1) in general settings. First the evolution may go through topological changes, and secondly the formula (1.2) does not hold for $\lambda$ in less than $C^{1, \alpha}$ settings. The first difficulty motivates us to study geometric properties that are preserved by the flow, and the second requires new ideas to obtain sufficient compactness to establish convergence to equilibrium.

In variational setting, (1.1) can be formulated based on its energy dissipation structure for the perimeter energy with volume preserving constraint. Using this structure [MSS16] and [Tak17] showed the existence of general distribution solution of (1.1). For our interest in geometric properties of solutions, we instead work with a modified version of viscosity solutions, where we consider an implicit choice of $\lambda$ so that the volume of the evolving set is preserved over time.

Motivated by recent results [MSS16] and [KK18, our strategy is to approximate (1.1) by the following flow as $\delta \rightarrow 0$ :

$$
\begin{cases}V & =-H+\lambda_{\delta}(t), \quad \lambda_{\delta}(t):=\gamma_{\delta}\left(\left|\Omega_{t}\right|\right) \text { on } \Gamma_{t}, \\ \Omega_{0}^{\delta} & =\Omega_{0}\end{cases}
$$

where $\gamma_{\delta}: \mathbb{R}^{+} \rightarrow \mathbb{R}$ for $\delta>0$ is defined by

$$
\gamma_{\delta}(s):=\frac{1}{\delta}(1-s)
$$

Let us mention that the comparison principle does not hold for both (1.1) and (1.3), so the notion of solutions should be understood as viscosity solutions with a priori given $\lambda_{\delta}(t)$ (see Definition 1.1 and Definition (2.6). Compared to the original flow (1.1), (1.3) holds an advantage that $\lambda_{\delta}(t)$ only depends on $\left|\Omega_{t}\right|$, thus it can be handled with little information on the regularity of $\Gamma_{t}$, which makes it easier to handle with viscosity solutions theory. The existence and uniqueness for viscosity solutions of (1.3) were proved in KK18. The following is summary of the main results in Theorem 3.1 \& 4.1 , Corollary 4.2 and Theorem 5.1

Key words and phrases. Mean curvature flow, viscosity solutions, minimizing movements, star-shaped, moving planes method, volume preserving mean curvature flow.

Inwon Kim was partially supported by NSF DMS-1566578.

Dohyun Kwon was partially supported by Kwanjeong Educational Foundation. 
Theorem 1. Let us denote $\mathcal{B}\left(x_{0}\right)$ to be the ball of unit volume centered at $x_{0}$. Under the geometric assumption on the initial data,

$$
\Omega_{0} \text { satisfies } \rho \text {-reflection (See Definition 2.1) for some } \rho \in\left[0,\left(c_{n} 5\right)^{-1}\right), c_{n}=\left|B_{1}(0)\right|^{1 / n} \text {. }
$$

There exists a viscosity solution $\left(\Omega_{t}^{\infty}, \lambda_{\infty}\right)$ of (1.1) approximated by solutions $\left\{\left(\Omega_{t}^{\delta}, \lambda_{\delta}\right)\right\}_{\delta>0}$ of (1.3) as $\delta>0$ with the following properties:

(a) Along a subsequence $\delta$ and for any finite time $T$, we have

$$
\max _{0 \leq t \leq T} d_{H}\left(\Omega_{t}^{\delta}, \Omega_{t}^{\infty}\right) \rightarrow 0, \lambda_{\delta} \rightarrow \lambda_{\infty} \text { in } L^{2}([0, T]) .
$$

(b) There exists $r>0$ such that for all $t, \delta>0$ both $\Omega_{t}^{\infty}$ and $\Omega_{t}^{\delta}$ contain the ball $B_{r}(0)$ and stay star-shaped with respect to it.

(c) $\left(\Omega_{t}^{\infty}\right)_{t \geq 0}$ uniformly converges to a ball of volume 1 , modulo translation, i.e.,

$$
\sup _{x_{0} \in \mathbb{R}^{n}} d_{H}\left(\Omega_{t}^{\infty}, \mathcal{B}\left(x_{0}\right)\right) \rightarrow 0 \text { as } t \rightarrow 0 .
$$

Let us briefly discuss the main ingredients and challenges in the context of literature.

Geometric properties Due to the low-dimensional nature of the flow, finite-time singularities even for smooth $\Omega_{0}$ can be expected in general. It is well known ( Hui87]) that convexity is preserved in the flow (1.1), and the global-time behavior of convex evolution, as well as exponential convergence to the unit ball, has been studied in the smooth case Hui87 and for anistropic flow And01 and BCCN09. Our goal in this paper is understanding the evolution of star-shaped sets. While it is suspected that star-shapedness is preserved in the evolution, it remains open to be proved. In KK18 we instead considered a stronger version of star-shapeness, i.e. the property $\rho$-reflection given in Section 2 , Roughly speaking this property amounts to the boundary of the set being Lipschitz with respect to the spherical coordinate given by $B_{\rho}(0)$. KK18 shows, by moving planes argument, that this property is preserved in the flow with volume-dependent forcing, which includes (1.3). In particular this property implies $(b)$ for $\Omega_{t}^{\delta}$, as well as an equi-continuity over time, yielding the first part of $(a)$. It should be pointed out that, as in KK18, our geometric arguments should be incorporated with the variational methods, since the underlying gradient flow structure of (1.1) and (1.3) provides both existence and asymptotic convergence results for both problems. For this reason our construction of solutions for (1.1)-(1.3) employs constrained minimizing movements with admissible sets only for star-shaped sets, which differs from the standard constructions.

Regularity of $\partial \Omega_{t}^{\infty}$ and Notions of solutions. To yield the second part of (a), we obtain uniform $L^{2}$ bound for $\lambda_{\delta}$, largely following the variational arguments in MSS16, adapted to our constrained minimizing movements described above. The main difficulty that is new in this paper is the lack of the uniform $L^{\infty}$ bound on $\lambda_{\infty}$. The bounds for $\lambda_{\delta}$ correlates to that of the total curvature $\int_{\partial \Omega_{t}} H d S$. An $L^{\infty}$ bound for $\lambda_{\infty}$ along with the geometric property of $\Omega_{t}$ would invoke parabolic regularity theory for curvature flows to yield smoothness of the flow, which in turn yields sufficient compactness to discuss the asymptotic behavior of the flow. Indeed this was the case for [KK18, where $\lambda_{\delta}$ in (1.3) is a priori bounded by $\frac{1}{\delta}$.

For convex case, Minkowski's quadratic inequality (See (78) in [BCCN09] and Proposition 4.1 in And01) yields a uniform bound on the total mean curvature of a set in terms of volume and perimeter. However, for non-convex set, this inequality fails and the total mean curvature can be unbounded (See Example A.1 and Example A.2.

For us there is only $L^{2}$ estimates are available on $\lambda_{\infty}$, which is inherited from $\lambda_{\delta}$ 's (see Section 4). For this reason, we fall short of obtaining regularity of $\partial \Omega_{t}^{\infty}$ that goes beyond Lipschitz. In particular this necessitates a notion of viscosity solutions of $V=-H+\lambda$ for a priori given $\lambda \in L_{\text {loc }}^{1}([0, \infty))$ (Definition 2.7). Moreover, to assert that the limit $\left(\Omega_{t}^{\infty}, \lambda_{\infty}\right)$ solves (1.1), our notion needs to stay stable under weak convergence of $\lambda$ in $L^{2}$. Once such notion is established for prescribed $\lambda$, we can introduce a notion of viscosity solutions of (1.1):

Definition 1.1. The pair $\left(\left(\Omega_{t}\right)_{t \geq 0}, \lambda\right)$ be a viscosity solution of (1.1) if $\left|\Omega_{t}\right|=\left|\Omega_{0}\right|$ and $\left(\Omega_{t}\right)_{t \geq 0}$ is a viscosity solution (See Definition 2.8) of $V=-H+\lambda(t)$. 
The extended notion for prescribed $\lambda$, Definition 2.7 enables us to analyze geometric properties of $\left(\left(\Omega_{t}\right)_{t \geq 0}, \lambda\right)$ for $\lambda \in L_{l o c}^{1}([0, \infty))$. Notions of viscosity solutions for time-integrable operator are previously introduced in [Ish85], Bou08a] and [Bou08b]. These previous notions however do not allow stability under weak convergence of operators, and thus in this aspect our notion is new. Our notions however coincide with the previous ones as a consequence of its stability properties, see Remark 2.16.

Note that gradient and curvature estimates of volume preserving mean curvature and classical mean curvature were proven in [Ath97] for rotationally symmetric case and [EH89] for entire graphs. We expect that this arguments with interior estimates from EH91 and Eck04 can be applied for our case with suitable modifications, but we do not pursue this issue here. With higher regularity, uniqueness of the solution for (1.1) may be shown by dilation arguments as in Gig06 and BCCN09.

Long-time behavior of the evolution As mentioned above, we are short of proving smoothness of $\Omega_{t}^{\infty}$ beyond its Lipschitz graph property, though we expect it to be true. Note that in non-smooth or nonconvex setting, perimeter difference may not converge into zero as Hausdorff distance converges to zero. This poses a challenge for proving asymptotic convergnce of $\Omega_{t}^{\infty}$. Our proof of perimeter convergence in the asymptotic limit uses both the uniform $L^{2}$ estimates of mean curvature and star-shapedness (See Lemma 5.3). ES98 and AKS10 show global well-posedness and exponential convergence if the initial condition is sufficiently close to a round sphere in Hölder norm and Sobolev norm. respectively. Similar results were proven for sufficiently small traceless second fundamental form of the initial condition in Li09. We mention that most of existing results on asymptotic convergence require regularity of the interface to be smoother than $C^{1, \alpha}$.

We finish this section with an outline of the paper. In section 2 we recall level set formulation of (1.1) and notions of the corresponding viscosity solutions for a prescribed and continuous $\lambda$. Then we extend the notion to $\lambda \in L_{l o c}^{1}([0, \infty))$ and establish its well-posedness by comparison principle. Then we are able to define the notion of solutions for our original flow (1.1) as given in Definition 1.1. In section 3 we introduce the approximation by (1.3) constructed by a constrained minimizing movement. Based on their geometric properties, we establish the first part of Theorem 1 (a) for the limiting set $\Omega_{\infty}$. Section 4 completes the statement of Theorem 1(a) and (b) by establishing a uniform $L^{2}$ bound of $\lambda_{\delta}$, using the variational construction of solutions for (1.3). This leads to the weak convergence of $\lambda_{\delta}$ to $\lambda_{\infty}$, While following the outline given by MSS16, our construction of local variation is more delicate (Lemma 4.7 and Lemma 4.8), since the perturbed set needs to stay within our geometric constraints. Finally in section 5 we prove Theorem 1(c), by establishing the perimeter convergence of $\Omega_{t}^{\infty}$ as $t \rightarrow \infty$, using the $L^{2}$ bound on $\lambda_{\infty}$ obtained in section 4 .

\section{Preliminaries ANd A NOtion of SOlution}

2.1. Notations. We begin with a list of definitions.

- $Q:=\mathbb{R}^{n} \times[0, \infty), \quad Q_{T}:=\mathbb{R}^{n} \times[0, T]$;

- $D_{r}\left(x_{0}, t_{0}\right):=B_{r}\left(x_{0}\right) \times\left(t_{0}-r^{2}, t_{0}\right], \quad \partial_{p} D_{r}:=\left(\bar{B}_{r}\left(x_{0}\right) \times\left\{t_{0}\right\}\right) \cup\left(\partial B_{r}\left(x_{0}\right) \times\left[t_{0}-r^{2}, t_{0}\right]\right)$;

- $C_{r, h}(x):=x+[-h, h] \times B_{r}^{n-1}(0), C_{r, h}^{+}(x):=x+[0, h] \times B_{r}^{n-1}(0), B_{r}^{n-1}(0):=\left\{x \in \mathbb{R}^{n-1},|x| \leq 1\right\} ;$

Next we recall some geometric properties from [FK14].

Definition 2.1. FK14, Definition 10] A bounded, open set $\Omega$ satisfies $\rho$-reflection if

(1) $\Omega$ contains $\overline{B_{\rho}(0)}$ and

(2) $\Omega$ satisfies that for all direction $\nu \in S^{n-1}$ and all $s>\rho$.

$$
\Psi_{\Pi_{\nu}(s)}\left(\Omega \cap \Pi_{\nu}^{+}(s)\right) \subset \Omega \cap \Pi_{\nu}^{-}(s)
$$

where $\Psi_{\Pi_{\nu}(s)}$ is a reflection function with respect to the hyperplane $\Pi_{\nu}(s):=\{x \cdot \nu=s\}$ defined by

$$
\Psi_{\Pi_{\nu}(s)}(x):=x-2\langle x-s \nu, \nu\rangle \nu, \quad \Pi_{\nu}^{+}(s):=\left\{x \in \mathbb{R}^{n}: x \cdot \nu>s\right\} \text { and } \Pi_{\nu}^{-}(s):=\left\{x \in \mathbb{R}^{n}: x \cdot \nu<s\right\} .
$$

Definition 2.2. A bounded set $\Omega$ in $\mathbb{R}^{n}$ is star-shaped with respect to a ball $B_{r}$ if for any point $y \in B_{r}$, $\Omega$ is star-shaped with respect to $y$. Let

$$
S_{r}:=\left\{\Omega: \text { star-shaped with respect to } B_{r}(0)\right\} \text { and } S_{r, R}:=S_{r} \cap\left\{\Omega: \Omega \subset B_{R}(0)\right\} .
$$


2.2. Preliminary notions of viscosity solutions. For $u: L \subset \mathbb{R}^{d} \rightarrow \mathbb{R}$ we denote its semi-continuous envelopes $u_{*}, u^{*}: \bar{L} \rightarrow \mathbb{R}$ by

$$
u_{*}(x):=\lim _{\epsilon \downarrow 0} \inf _{\substack{|x-y|<\epsilon, y \in L}} u(y) \quad \text { and } u^{*}(x):=\lim _{\epsilon \downarrow 0} \sup _{\substack{|x-y|<\epsilon, y \in L}} u(y) .
$$

For a sequence of functions $\left\{u_{k}\right\}_{k \in \mathbb{N}}$ on $Q$,

$$
\begin{aligned}
\limsup _{k \rightarrow \infty} u_{k}(x, t) & :=\lim _{j \rightarrow \infty} \sup \left\{u_{k}(y, s): k \geq j, \quad|y-x| \leq \frac{1}{j}, \quad|s-t| \leq \frac{1}{j}\right\}, \\
\liminf _{k \rightarrow \infty} u_{k}(x, t) & :=\lim _{j \rightarrow \infty} \inf \left\{u_{k}(y, s): k \geq j, \quad|y-x| \leq \frac{1}{j}, \quad|s-t| \leq \frac{1}{j}\right\} .
\end{aligned}
$$

In the level set formation, $\Omega_{t}$ is given by $\Omega_{t}(u):=\left\{x \in \mathbb{R}^{n}: u(x, t)>0\right\}$ where $u: Q \rightarrow \mathbb{R}$ solves the following equation:

$$
u_{t}=F\left(D u, D^{2} u\right)+\lambda(t)|D u|
$$

where $F:\left(\mathbb{R}^{n} \backslash\{0\}\right) \times \mathcal{S}^{n \times n} \rightarrow \mathbb{R}$ is given by

$$
F(p, X):=\operatorname{trace}\left(\left(I-\frac{p}{|p|} \otimes \frac{p}{|p|}\right) X\right) .
$$

with initial data

$$
u(x, 0)=u_{0}(x):=\chi_{\Omega_{0}}-\chi_{\Omega_{0}^{C}} \text { for } x \in \mathbb{R}^{n} .
$$

Let us recall definitions of viscosity solutions of (2.6) and (1.3) with fixed $\lambda \in C\left(\mathbb{R}_{0}^{+}\right)$.

Definition 2.3. [CGG91, Definition 2.1], Bar13, Definition 6.1]

- A function $u: Q \rightarrow \mathbb{R}$ is a viscosity subsolution of (2.6) if $u^{*}<\infty$ and for any $\phi \in \mathcal{C}^{2,1}(Q)$ that touches $u^{*}$ from above at $\left(x_{0}, t_{0}\right)$ we have

$$
\phi_{t}\left(x_{0}, t_{0}\right) \leq F^{*}\left(D \phi\left(x_{0}, t_{0}\right), D^{2} \phi\left(x_{0}, t_{0}\right)\right)+\lambda\left(t_{0}\right)\left|D \phi\left(x_{0}, t_{0}\right)\right| .
$$

where $F$ is given in (2.7). Also, $u^{*}$ and $F^{*}$ are given in (2.3).

- A function $u: Q \rightarrow \mathbb{R}$ is a viscosity supersolution of (2.6) if $u_{*}>-\infty$ and for any $\phi \in \mathcal{C}^{2,1}(Q)$ that touches $u_{*}$ from below at $\left(x_{0}, t_{0}\right)$ we have

$$
\phi_{t}\left(x_{0}, t_{0}\right) \geq F_{*}\left(D \phi\left(x_{0}, t_{0}\right), D^{2} \phi\left(x_{0}, t_{0}\right)\right)+\lambda\left(t_{0}\right)\left|D \phi\left(x_{0}, t_{0}\right)\right| .
$$

where $F$ is given in (2.7). Also, $u_{*}$ and $F_{*}$ are given in (2.3).

- A function $u: Q \rightarrow \mathbb{R}$ is a viscosity solution of (2.6)-(2.8) (or (2.51) ) if $u^{*}$ is a viscosity subsolution of (2.6) and $u_{*}$ is a viscosity supersolution of (2.6), and if $u^{*}=\left(u_{0}\right)^{*}$ and $u_{*}=\left(u_{0}\right)_{*}$ at $t=0$.

- For any $\lambda \in C\left(\mathbb{R}_{0}^{+}\right),\left(\Omega_{t}\right)_{t \geq 0}$ be a viscosity solution (subsolution or supersolution, respectively) of

$$
V=-H+\lambda(t)
$$

if $u:=\chi_{\Omega_{t}}-\chi_{\Omega_{t}}$ is a viscosity solution (subsolution or supersolution, respectively) of (2.6)-(2.8).

We also recall definitions of classical solutions and equivalent definitions of viscosity solutions of (2.6) with fixed $\lambda \in C\left(\mathbb{R}_{0}^{+}\right)$.

Definition 2.4. Consider a cylinder $D_{r} \subset Q$ and $F$ given in (2.7).

- A function $\phi \in C^{2,1}\left(D_{r}\right)$ is a classical subsolution in $D_{r}$ of (2.6) if it holds that

$$
\phi_{t} \leq F_{*}\left(D \phi, D^{2} \phi\right)+\lambda|D \phi| \text { in } D_{r} .
$$

- A function $\phi \in C^{2,1}\left(D_{r}\right)$ is a classical supersolution in $D_{r}$ of (2.6) if it holds that

$$
\phi_{t} \geq F^{*}\left(D \phi, D^{2} \phi\right)+\lambda|D \phi| \text { in } D_{r} .
$$

- We say that $\phi \in C^{2,1}\left(D_{r}\right)$ is a classical strict subsolution (supersolution, respectively) on $D_{r}$ of (2.6) if the strict inequality of (2.11) (2.12), respectively) holds in $D_{r}$ 
Definition 2.5. [CSS05, Definition 7.2]

- A function $u: Q \rightarrow \mathbb{R}$ is a viscosity subsolution of (2.6) if $u^{*}<\infty$ and for $D_{r} \subset Q$ and for every classical strict supersolution $\phi \in C^{2,1}\left(D_{r}\right), u^{*}<\phi$ on $\partial_{p} D_{r}$ implies $u^{*}<\phi$ in $\bar{Q}$.

- A function $u: Q \rightarrow \mathbb{R}$ is a viscosity supersolution of (2.6) if $u_{*}>-\infty$ and $D_{r} \subset Q$ and for every classical strict subsolution $\phi \in C^{2,1}\left(D_{r}\right), u_{*}>\phi$ on $\partial_{p} D_{r}$ implies $u_{*}>\phi$ in $\bar{Q}$.

Definition 2.6. [KK18, Definition 2.7] For $\lambda \in C\left(\mathbb{R}_{0}^{+}\right),\left(\left(\Omega_{t}\right)_{t \geq 0}, \lambda\right)$ be a viscosity solution of

$$
V=-H+\gamma\left(\left|\Omega_{t}\right|\right)
$$

where $\gamma: \mathbb{R}_{0}^{+} \rightarrow \mathbb{R}$ if $\left(\Omega_{t}\right)_{t \geq 0}$ is a viscosity solution of (2.10) with $\gamma\left(\left|\Omega_{t}\right|\right)=\lambda(t)$.

2.3. Viscosity Solutions for $L_{l o c}^{1}$ forcing. In this section, we develop a notion of viscosity solutions for (2.6) for a fixed $\lambda$ in $L_{\text {loc }}^{1}([0, \infty))$. Some notations are in order. For $\gamma \in C\left(\mathbb{R}_{0}^{+}\right)$, the sup convolution $\widehat{u}(\cdot ; \gamma)$ and inf convolution $\widetilde{u}(\cdot ; \gamma)$ is given by

$$
\begin{aligned}
& \widehat{u}(x, t ; \gamma):=\sup _{y \in \bar{B}_{\gamma(t)}(x)} u(y, t), \\
& \widetilde{u}(x, t ; \gamma):=\inf _{y \in \bar{B}_{\gamma(t)}(x)} u(y, t) .
\end{aligned}
$$

Note that $\widehat{u^{*}}=(\widehat{u})^{*}$ and $\widetilde{u_{*}}=(\widetilde{u})_{*}$.

Definition 2.7. For $\lambda \in L_{l o c}^{1}([0, \infty)), \Lambda(t):=\int_{0}^{t} \lambda(s) d s$ and $F$ given in (2.7),

- A function $u: Q \rightarrow \mathbb{R}$ is a viscosity subsolution of (2.6) if $u^{*}<\infty$ and for any $0 \leq t_{1}<t_{2}$ and $\Theta \in \mathcal{C}^{1}\left(\left(t_{1}, t_{2}\right)\right) \cap \mathcal{C}\left(\left[t_{1}, t_{2}\right]\right)$ such that $\Theta \geq \Lambda$ in $\left[t_{1}, t_{2}\right]$, a function $\widehat{u}=\widehat{u}(\cdot ; \Theta-\Lambda)$ given in (2.14) is a viscosity subsolution of

$$
u_{t}=F\left(D u, D^{2} u\right)+\Theta^{\prime}|D u|
$$

in $\left(t_{1}, t_{2}\right) \times \mathbb{R}^{n}$ in the sense of Definition 2.3 .

- A function $u: Q \rightarrow \mathbb{R}$ is a viscosity supersolution of (2.6) if $u_{*}>-\infty$ and for any $0 \leq t_{1}<t_{2}$ and $\Theta \in \mathcal{C}^{1}\left(\left(t_{1}, t_{2}\right)\right) \cap \mathcal{C}\left(\left[t_{1}, t_{2}\right]\right)$ such that $\Theta \leq \Lambda$ in $\left[t_{1}, t_{2}\right]$, a function $\widetilde{u}=\widetilde{u}(\cdot ;-\Theta+\Lambda)$ given in (2.15) is a viscosity supersolution of (2.16) in $\left(t_{1}, t_{2}\right) \times \mathbb{R}^{n}$ in the sense of Definition 2.3.

- A function $u: Q \rightarrow \mathbb{R}$ is a viscosity solution of (2.6)-(2.8) (or (2.51)) if $u^{*}$ is a viscosity subsolution of (2.6) and $u_{*}$ is a viscosity supersolution of (2.6), and if $u^{*}=\left(u_{0}\right)^{*}$ and $u_{*}=\left(u_{0}\right)_{*}$ at $t=0$.

Note that if $\lambda$ is continuous, then our definition coincides the usual definition. We also define the corresponding notion of viscosity solutions for sets.

Definition 2.8. For $\lambda \in L_{l o c}^{1}([0, \infty))$, let $\left(\Omega_{t}\right)_{t \geq 0}$ be a viscosity solution (subsolution or supersolution, respectively) of $V=-H+\lambda(t)$ if $u:=\chi_{\Omega_{t}}-\chi_{\Omega_{t}}{ }^{c}$ is a viscosity solution (subsolution or supersolution, respectively) of (2.6)-(2.8).

Recall that the definition of solutions for (1.1), is based on above definition.

Definition 1.1. For $\lambda \in L_{\text {loc }}^{1}([0, \infty))$, the pair $\left(\left(\Omega_{t}\right)_{t \geq 0}, \lambda\right)$ be a viscosity solution of (1.1) if $\left|\Omega_{t}\right|=\left|\Omega_{0}\right|$ and $\left(\Omega_{t}\right)_{t \geq 0}$ is a viscosity solution of $V=-H+\lambda(t)$.

Remark 2.9. Note that for $\lambda \in \mathcal{C}\left(\mathbb{R}_{0}^{+}\right)$, this definition coincides with Definition 2.3. First of all, KK18, Lemma 2.5] implies that a viscosity subsolution (supersolution, respectively) in the sense of Definition 2.3 is that in the sense of Definition 2.7. On the other hand, if $\lambda \in \mathcal{C}\left(\mathbb{R}_{0}^{+}\right)$, then $\Lambda \in \mathcal{C}^{1}\left(\mathbb{R}_{0}^{+}\right)$. Thus, we can choose $\Theta=\Lambda$. As $\widehat{u}(\cdot ; 0)=\widetilde{u}(\cdot ; 0)=u$ in $Q$, we conclude that a viscosity subsolution (supersolution, respectively) in the sense of Definition 2.7 is that in the sense of Definition 2.3 .

In the rest of this section, we develop existence and uniqueness results for (2.6). We first show the comparison principle in Theorem 2.10, which yields uniqueness (Corollary 2.11). Moreover, we show the stability of viscosity solutions of $V=-H+\lambda_{k}(t)$ for $\left\{\lambda_{k}\right\}_{k \in \mathbb{N}} \subset L_{l o c}^{1}([0, \infty))$ when a sequence of time integrals of $\lambda_{k}$ converges. This yields existence (Corollary 2.15). 
Theorem 2.10. Let $u$ and $v$ be a viscosity subsolution and supersolution of (2.6), respectively, in the sense of Definition 2.7. If for some $r>0$ and $\left(x_{0}, t_{0}\right) \in Q$ we have $u^{*} \leq v_{*}$ on $\partial_{p} D_{r}\left(x_{0}, t_{0}\right)$, then

$$
u^{*} \leq v_{*} \text { on } D_{r}\left(x_{0}, t_{0}\right) \text {. }
$$

Proof. For simplicity, consider $\left(x_{0}, t_{0}\right)=\left(0, r^{2}\right)$ and denote $D_{r}:=D_{r}\left(0, r^{2}\right)=B_{r}(0) \times\left(0, r^{2}\right]$. Note that we may assume the following, by adding a small constant to $v$ :

$$
u^{*}<v_{*} \text { on } \partial_{p} D_{r}\left(x_{0}, t_{0}\right) \text {. }
$$

1. Let us show that there exists $\varepsilon_{1}>0$ such that

$$
\widehat{u}^{*}\left(\cdot ; \varepsilon_{1}\right)<\widetilde{v}_{*}\left(\cdot ; \varepsilon_{1}\right) \text { on } \partial_{p} D_{r} .
$$

Suppose that (2.19) does not hold for all $\varepsilon_{1}>0$. Then, there exists a sequence $\left\{x_{k}\right\}_{k \in \mathbb{N}} \subset \partial_{p} D_{r}$ such that

$$
\widehat{u}^{*}\left(x_{k} ; \frac{1}{k}\right) \geq \widetilde{v}_{*}\left(x_{k} ; \frac{1}{k}\right) .
$$

By the semi-continuity of $u^{*}$ and $v_{*}$, there exists $\left\{\left(y_{k}, z_{k}\right)\right\}_{k \in \mathbb{N}}$ such that

$$
\left|x_{k}-y_{k}\right| \leq \frac{1}{k}, \quad\left|x_{k}-z_{k}\right| \leq \frac{1}{k}
$$

and

$$
u^{*}\left(y_{k}\right) \geq v_{*}\left(z_{k}\right)
$$

By compactness of $\bar{D}_{r+1}$, there exists a subsequence $\left\{k_{i}\right\}_{i \in \mathbb{N}}$ and $\left(y^{*}, z^{*}\right)$ such that $\left\{\left(y_{k_{i}}, z_{k_{i}}\right)\right\}_{k_{i} \in \mathbb{N}}$ converges to $\left(y^{*}, z^{*}\right)$. From (2.21) and the closedness of $\partial_{p} D_{r}$, we conclude that $y^{*}=z^{*} \in \partial_{p} D_{r}$. From (2.22) and the semi-continuity of $u^{*}$ and $v_{*}$, it holds that

$$
u^{*}\left(y^{*}\right) \geq \limsup _{i \rightarrow \infty} u^{*}\left(y_{k_{i}}\right) \geq \liminf _{i \rightarrow \infty} v_{*}\left(z_{k_{i}}\right) \geq v_{*}\left(z^{*}\right)=v_{*}\left(y^{*}\right)
$$

This contradicts to (2.18).

2. Note that $\mathcal{C}^{1}\left(\left[0, r^{2}\right]\right)$ is dense in $\mathcal{C}\left(\left[0, r^{2}\right]\right)$. There exists $\Theta \in \mathcal{C}^{1}\left(\left[0, r^{2}\right]\right)$ such that

$$
\sup _{t \in\left[0, r^{2}\right]}|\Lambda(t)-\Theta(t)| \leq \frac{\varepsilon_{1}}{2} .
$$

where $\varepsilon_{1}>0$ is given in Step 1 . Then, $\widehat{u}^{*}\left(\cdot ; \frac{\varepsilon_{1}}{2}+\Theta(t)-\Lambda(t)\right)$ and $\widetilde{v}_{*}\left(\cdot ; \frac{\varepsilon_{1}}{2}-\Theta(t)+\Lambda(t)\right)$ are well-defined in $D_{r}$. Note that $\widehat{u}^{*}$ and $\widetilde{v}_{*}$ given above are respectively viscosity subsolution and supersolutions of (2.16).

From (2.24) and (2.19), it holds that

$$
\widehat{u}^{*}\left(\cdot ; \frac{\varepsilon_{1}}{2}+\Theta(t)-\Lambda(t)\right) \leq \widehat{u}^{*}\left(\cdot ; \varepsilon_{1}\right)<\widetilde{v}_{*}\left(\cdot ; \varepsilon_{1}\right) \leq \widetilde{v}_{*}\left(\cdot ; \frac{\varepsilon_{1}}{2}-\Theta(t)+\Lambda(t)\right) .
$$

on $\partial_{p} D_{r}$. From comparison principle for (2.16) in CGG91, Theorem 4.1], we conclude that

$$
\widehat{u}^{*}\left(\cdot ; \frac{\varepsilon_{1}}{2}+\Theta(t)-\Lambda(t)\right)<\widetilde{v}_{*}\left(\cdot ; \frac{\varepsilon_{1}}{2}-\Theta(t)+\Lambda(t)\right) .
$$

on $D_{r}$, which implies (2.17).

Corollary 2.11. For $g \in \mathcal{C}\left(\partial_{p} D_{r}\right)$, there is at most one viscosity solution $u$ of (2.6) with $u^{*}=u_{*}=g$ on $\partial_{p} D_{r}$ in the sense of Definition 2.7.

Next we develop stability results for $\left\{\lambda_{k}\right\}_{k \in \mathbb{N}}$ such that $\left\{\Lambda_{k}\right\}_{k \in \mathbb{N}}$ uniformly converges to $\Lambda_{\infty}$ where

$$
\left\{\lambda_{k}\right\}_{k \in \mathbb{N} \cup\{+\infty\}} \subset L_{l o c}^{1}([0, \infty)) \text { and } \Lambda_{k}(t):=\int_{0}^{t} \lambda_{k}(s) d s \text { for } k \in \mathbb{N} \cup\{+\infty\} .
$$

Note that this gives stability results for weak convergence of $\left\{\lambda_{k}\right\}_{k \in \mathbb{N}} \subset L^{p}([0, T])$ for any $p \in(1, \infty]$. This results will be used Corollary 4.2 
Theorem 2.12. For $\left\{\lambda_{k}\right\}_{k \in \mathbb{N} \cup\{+\infty\}}$ and $\left\{\Lambda_{k}\right\}_{k \in \mathbb{N} \cup\{+\infty\}}$ given in (2.27), assume that $\left\{\Lambda_{k}\right\}_{k \in \mathbb{N}}$ locally uniformly converges to $\Lambda_{\infty}$. Let $\left\{u_{k}\right\}_{k \in \mathbb{N}}$ be a sequence of viscosity subsolutions (supersolutions, respectively) of (2.6) with $\lambda=\lambda_{k}$ for all $k \in \mathbb{N}$. If $u:=\limsup _{k \rightarrow \infty}^{*} u_{k}<\infty \quad\left(u:=\liminf _{k \rightarrow \infty} u_{k}>-\infty\right.$, respectively), then $u$ is a viscosity subsolution (supersolutions) of (2.6) with $\lambda=\lambda_{\infty}$ in the sense of Definition 2.7.

Proof. We only show the subsolution part, since the rest can be shown with parallel arguments. Let $\left\{u_{k}\right\}_{k \in \mathbb{N}}$ be a sequence of viscosity subsolutions.

1. Choose any $0 \leq t_{1}<t_{2}$ and $\Theta \in \mathcal{C}^{1}\left(\left(t_{1}, t_{2}\right)\right) \cap \mathcal{C}\left(\left[t_{1}, t_{2}\right]\right)$ such that $\Theta \geq \Lambda_{\infty}$ in $\left[t_{1}, t_{2}\right]$. Let us show that $\widehat{u}\left(\cdot ; \Theta-\Lambda_{\infty}\right)$ given in (2.14) is a viscosity subsolution of (2.16). From the equivalent definition of viscosity solutions in Definition 2.5, it is enough to show that for any $D_{r} \subset Q$

$$
\widehat{u}^{*}\left(\cdot ; \Theta-\Lambda_{\infty}\right)<\phi \text { in } D_{r}
$$

where $\phi \in \mathcal{C}^{2,1}\left(D_{r}\right)$ is a classical strict supersolution of (2.16) given in Definition 2.4 such that

$$
\widehat{u}^{*}\left(\cdot ; \Theta-\Lambda_{\infty}\right)<\phi \text { on } \partial_{p} D_{r} .
$$

First, as $u<+\infty$ and $u$ is upper semicontinuous, we get $\widehat{u}^{*}<\infty$. Next, by the upper semicontinuity of $u^{*}$, there exists $\varepsilon_{2}>0$ such that

$$
\widehat{u}^{*}\left(\cdot ; \Theta-\Lambda_{\infty}\right)<\phi-3 \varepsilon_{2} \quad \text { on } \partial_{p} D_{r} .
$$

From the upper semicontinuity again, there exists $\varepsilon_{1}>0$ such that

$$
\widehat{u}^{*}\left(\cdot ; \varepsilon_{1}+\Theta-\Lambda_{\infty}\right)<\phi-2 \varepsilon_{2} \quad \text { on } \partial_{p} D_{r} .
$$

By uniform convergence of $\Lambda_{k}$, there exists $k_{1} \in \mathbb{N}$ such that for all $k>k_{1}$, it holds that

$$
\left|\Lambda_{\infty}-\Lambda_{k}\right|<\frac{\varepsilon_{1}}{2}
$$

in $\left[t_{1}, t_{2}\right]$. By definition, $\widehat{u}_{k}=\widehat{u}_{k}\left(\cdot ; \varepsilon_{1}+\Theta-\Lambda_{k}\right)$ is a viscosity subsolutions of (2.16) in $\left(t_{1}, t_{2}\right)$ for all $k>k_{1}$.

2. Let us show that there exists $k_{2} \in \mathbb{N}$ such that $k_{2}>k_{1}$ and

$$
\widehat{u}_{k}^{*}\left(\cdot ; \varepsilon_{1}+\Theta-\Lambda_{k}\right)<\phi-\varepsilon_{2} \text { on } \partial_{p} D_{r} \text { for all } k \geq k_{2} .
$$

where $k_{1}$ is given in Step 1. Suppose that such $k_{2}$ does not exist. Then, there exists a sequence $\left\{k_{i}\right\}_{i \in \mathbb{N}}$ converging to infinity and $\left\{x_{k_{i}}\right\}_{i \in \mathbb{N}} \subset \partial_{p} D_{r}$ such that $k_{i} \geq k_{1}$ and

$$
\widehat{u}_{k_{i}}^{*}\left(x_{k_{i}} ; \varepsilon_{1}+\Theta-\Lambda_{k_{i}}\right) \geq \phi\left(x_{k_{i}}\right)-\varepsilon_{2} \text { on } \partial_{p} D_{r} \text { for all } i \in \mathbb{N} \text {. }
$$

By the upper semi-continuity of $u^{*}$, there exists $\left\{y_{k_{i}}\right\}_{i \in \mathbb{N}}$ such that

$$
\left|y_{k_{i}}-x_{k_{i}}\right| \leq \varepsilon_{1}+\Theta-\Lambda_{k_{i}} \quad \text { and } u_{k_{i}}^{*}\left(y_{k_{i}}\right) \geq \phi\left(x_{k_{i}}\right)-\varepsilon_{2}
$$

Furthermore, there exists $\left\{z_{k_{i}}\right\}_{i \in \mathbb{N}}$ such that

$$
\left|z_{k_{i}}-y_{k_{i}}\right| \leq \frac{1}{k_{i}} \quad \text { and } u_{k_{i}}\left(z_{k_{i}}\right)+\varepsilon_{2} \geq u_{k_{i}}^{*}\left(y_{k_{i}}\right)
$$

From (2.35) and (2.36), we get

$$
\left|z_{k_{i}}-x_{k_{i}}\right| \leq \varepsilon_{1}+\Theta-\Lambda_{k_{i}}+\frac{1}{k_{i}} \text { and } u_{k_{i}}\left(z_{k_{i}}\right) \geq \phi\left(x_{k_{i}}\right)-2 \varepsilon_{2}
$$

As $\left\{x_{k_{i}}\right\}_{i \in \mathbb{N}} \subset \partial_{p} D_{r}$, (2.32) and (2.37) imply that

$$
\left\{z_{k_{i}}\right\}_{i \in \mathbb{N}} \subset \bar{D}_{\widehat{r}} \text { where } \widehat{r}=r+2 \varepsilon_{1}+\left\|\Theta-\Lambda_{\infty}\right\|_{\infty}+1 .
$$

From compactness of $\bar{D}_{\widehat{r}}$, there exists a subsequence $\left\{k_{i_{j}}\right\}_{i \in \mathbb{N}}$ and $\left(x^{*}, z^{*}\right)$ such that $\left\{\left(x_{k_{i_{j}}}, z_{k_{i_{j}}}\right)\right\}_{j \in \mathbb{N}}$ converges to $\left(x^{*}, z^{*}\right)$. (2.37) implies that

$$
\left|z^{*}-x^{*}\right| \leq \varepsilon_{1}+\Theta-\Lambda_{\infty}
$$

and

$$
u\left(z^{*}\right) \geq \limsup _{j \rightarrow \infty} u_{k_{i_{j}}}\left(z_{k_{i_{j}}}\right) \geq \limsup _{j \rightarrow \infty} \phi\left(x_{k_{i_{j}}}\right)-2 \varepsilon_{2}=\phi\left(x^{*}\right)-2 \varepsilon_{2}
$$

This contradicts to (2.31) and we conclude (2.33). 
3. From Step 1 and (2.33), comparison principle in Theorem 2.10 implies that

$$
\widehat{u}_{k}^{*}\left(\cdot ; \varepsilon_{1}+\Theta-\Lambda_{k}\right)<\phi-\varepsilon_{2} \text { in } D_{r} \text { for all } k \geq k_{2} \text {. }
$$

where $\varepsilon_{1}$ and $\varepsilon_{2}$ are given in (2.31), and $k_{2}$ is given in (2.33). The above and (2.32) imply that

$$
u_{k}(y)<\phi(x)-\varepsilon_{2} \text { for all } x \in D_{r} \text { and } y \in B_{\frac{\varepsilon_{1}}{2}+\Theta-\Lambda_{\infty}}(x) \text { for all } k \geq k_{2} \text {. }
$$

and we conclude (2.28).

Let us construct radial barriers of (2.6).

Lemma 2.13. For $\Lambda: \mathbb{R}_{0}^{+} \rightarrow \mathbb{R}$ given by

$$
\Lambda(t):=\int_{0}^{t} \lambda(s) d s
$$

and $c \in \mathbb{R}$, define $\zeta^{-}: Q \rightarrow \mathbb{R}$ and $\zeta^{+}: Q \rightarrow \mathbb{R}$ by

$$
\zeta^{-}(x, t ; \Lambda, c):=-\chi_{\left\{x \in \mathbb{R}^{n}:|x|<c-\Lambda(t)\right\}}(x) \text { and } \quad \zeta^{+}(x, t ; \Lambda, c):=\chi_{\left\{x \in \mathbb{R}^{n}:|x|<c+\Lambda(t)\right\}}(x) .
$$

Then, $\zeta^{-}$and $\zeta^{+}$are a viscosity subsolution and supersolution, respectively, of (2.6) in the sense of Definition 2.7.

Proof. Let us show that $\zeta^{-}$is a viscosity subsolution of (2.6) only. The respective one can be shown by parallel arguments.

Choose any $0 \leq t_{1}<t_{2}$ and $\Theta \in \mathcal{C}^{1}\left(\left(t_{1}, t_{2}\right)\right) \cap \mathcal{C}\left(\left[t_{1}, t_{2}\right]\right)$ such that $\Theta \geq \Lambda$ in $\left[t_{1}, t_{2}\right]$. Let us show that $\widehat{\zeta^{-}}(\cdot ; \Theta-\Lambda)$ given in (2.14) is a viscosity subsolution of (2.16). Note that we have

$$
\widehat{\zeta^{-}}(x, t ; \Theta-\Lambda)=-\chi_{\mathcal{N}_{t}}(x) \text { where } \mathcal{N}_{t}:=\left\{x \in \mathbb{R}^{n}:|x|<c-\Theta(t)\right\}
$$

in $Q$.

Suppose that $\phi \in \mathcal{C}^{2,1}(Q)$ touches $\widehat{\zeta^{-}}$from above at $\left(x_{0}, t_{0}\right)$. First, consider the case $\left|x_{0}\right| \neq c-\Theta\left(t_{0}\right)$. In this case, as $\mathcal{N}_{t}$ given in (2.45) moves continuously in time, $\widehat{\zeta^{-}}$is constant near $\left(x_{0}, t_{0}\right)$. Thus, it holds that

$$
\phi_{t}\left(x_{0}, t_{0}\right) \leq 0, \quad D \phi\left(x_{0}, t_{0}\right)=0, \text { and } D^{2} \phi\left(x_{0}, t_{0}\right) \geq 0 .
$$

The ellipticity of $F$ given in (2.7) and (2.46) implies

$$
\phi_{t}\left(x_{0}, t_{0}\right) \leq F^{*}\left(D \phi\left(x_{0}, t_{0}\right), D^{2} \phi\left(x_{0}, t_{0}\right)\right)+\Theta^{\prime}\left(t_{0}\right)\left|D \phi\left(x_{0}, t_{0}\right)\right| .
$$

Let us consider the case $\left|x_{0}\right|=c-\Theta\left(t_{0}\right)$. If either $x_{0}=0$ or $x_{0}$ is a local minimum of $\phi\left(\cdot, t_{0}\right)$, then by the parallel arguments above, we get (2.46) and (2.47). Otherwise, both $\mathcal{N}_{t}$ given in (2.45) and a sublevel set $O_{t}$ of $\phi$ defined by

$$
O_{t}:=\left\{x \in \mathbb{R}^{n}: \phi(x, t)<\phi\left(x_{0}, t_{0}\right)\right\}
$$

are nonempty near $\left(x_{0}, t_{0}\right)$. By comparing the normal velocity and mean curvature of the level sets $\mathcal{N}_{t}$ and $O_{t}$, we conclude that

$$
\frac{\phi_{t}}{|D \phi|}\left(x_{0}, t_{0}\right) \leq \Theta^{\prime}\left(t_{0}\right) \text { and } \nabla \cdot\left(\frac{D \phi}{|D \phi|}\right)\left(x_{0}, t_{0}\right) \geq \frac{n-1}{\left|x_{0}\right|}>0 .
$$

which implies (2.47).

Let us recall $\mathcal{C}_{a}$ from [CGG91] for $\mathcal{N} \subset \mathbb{R}^{k}, k \in \mathbb{N}$ and $a \in \mathbb{R}$,

$$
\mathcal{C}_{a}(\mathcal{N}):=\{g \in \mathcal{C}(\mathcal{N}): g-a \text { has compact support in } \mathcal{N}\}
$$

and consider continuous initial data $g \in \mathcal{C}_{a}\left(\mathbb{R}^{n}\right)$,

$$
u(x, 0)=u_{0}(x):=g(x) \text { for } x \in \mathbb{R}^{n} .
$$

such that $\left\{x \in \mathbb{R}^{n}: g(x)>0\right\}=\Omega_{0}$ and $\left\{x \in \mathbb{R}^{n}: g(x)<0\right\}=\left(\Omega_{0}\right)^{C}$. 
From Theorem 2.10 and Theorem 2.12 combining with radial barriers in Lemma 2.13, we get existence and uniqueness of (2.6) with continuous initial data.

Theorem 2.14. For $T>0$, there is a unique viscosity solution $u$ in $\mathcal{C}_{a}\left(Q_{T}\right)$ of $(2.6)-(2.51)$ in the sense of Definition 2.7

Proof. As $\mathcal{C}^{1}([0, T])$ is dense in $\mathcal{C}([0, T])$, there exists $\left\{\Theta_{k}\right\}_{k \in \mathbb{N}} \subset \mathcal{C}^{1}([0, T])$ such that $\left\{\Theta_{k}\right\}_{k \in \mathbb{N}}$ uniformly converges to $\Lambda$ in $[0, T]$. From the existence of viscosity solutions in [CGG91, Theorem 6.8] of

$$
u_{t}=F\left(D u, D^{2} u\right)+\left(\Theta_{k}\right)^{\prime}|D u| \text { in } Q
$$

with initial data (2.51), there exists a sequence of viscosity solutions $\left\{u_{k}\right\}_{k \in \mathbb{N}} \subset \mathcal{C}_{a}\left(Q_{T}\right)$ of (2.52)-(2.51). Here, $F$ and $\mathcal{C}_{a}$ are given in (2.7) and (2.50), respectively.

Define $u^{+}:=\lim \sup ^{*} u_{k}$ and $u^{-}:=\liminf _{*} u_{k}$. As $g \in \mathcal{C}_{a}\left(\mathbb{R}^{n}\right)$, Theorem 2.10 implies $\left\|u_{k}\right\|_{L^{\infty}} \leq$ $\|g\|_{L^{\infty}}$ and thus $\left\|u^{ \pm}\right\|_{L^{\infty}}<+\infty$. Furthermore, by comparing $\left\{u_{k}\right\}_{k \in \mathbb{N}}$ with radial barriers $\|g\|_{L^{\infty}} \zeta^{ \pm}\left(\cdot ; \Theta_{k}, c\right)$ given in Lemma 2.13 for sufficiently large $c>0$, we conclude that the supports of $\left\{u_{k}\right\}_{k \in \mathbb{N}}$ are uniformly bounded in $Q_{T}$ for all $k \in \mathbb{N}$. Thus, $u^{ \pm}-a$ have compactly supports in $Q_{T}$.

Let us show that

$$
u^{+}=u^{-} \quad \text { in } Q_{T} .
$$

First, by definition of lim sup* and lim $\inf _{*}$ in (2.4), it holds that

$$
u^{+} \geq u^{-} \quad \text { in } Q_{T} \text {. }
$$

On the other hand, from the uniform convergence of $\left\{\Theta_{k}\right\}_{k \in \mathbb{N}}$ and Theorem 2.12, $u^{+}$and $u^{-}$are a viscosity subsolution and supersolution of (2.6)-(2.51), respectively. As $\left(u^{+}\right)^{*}=\left(u^{-}\right)_{*}=g$ at $t=0$, Theorem 2.10 implies

$$
u^{+} \leq u^{-} \quad \text { in } Q_{T} .
$$

Therefore, we get (2.53) from (2.54) and (2.55). From Corollary 2.11, we conclude that $u^{+}\left(=u^{-}\right)$is a unique viscosity solution in $\mathcal{C}_{a}\left(\mathbb{R}^{n}\right)$ of (2.6)-(2.51).

From parallel arguments in [BSS93, Theorem 2.1], we conclude existence of (2.6)-(2.8).

Corollary 2.15. There exists a unique viscosity solution of (2.6)-(2.8) in the sense of Definition 2.7.

Remark 2.16. As a consequence of Theorem 2.12 and Theorem 2.14 we conclude that our notion in Definition 2.7 coincides with viscosity solutions in Bou08a. This can be shown by smooth approximations of the operator and stability of each notions under strong $L^{1}$-convergence of forcing term.

\section{Approximation of Volume Preserving Mean Curvature Flow}

In this section, we construct a solution of (2.6) by (1.3). We show that viscosity solutions $\left(\Omega_{t}^{\delta}\right)_{t>0}$ of (1.3) are equicontinuous in Hausdorff distance, based on the geometric properties of $\Omega_{t}^{\delta}$. This yields uniform convergence of $\Omega_{t}^{\delta}$ in Hausdorff distance in Theorem 3.1. We will conclude in Section 4 that the limit of this strong convergence is a viscosity solution of (1.1). While much of the results in this section follows that of KK18, our focus here to obtain uniform estimates that stay independent of $\delta>0$ as $\delta \rightarrow 0$.

Here is main theorem of this section.

Theorem 3.1. There exists a sequence $\left\{\delta_{i}\right\}_{i \in \mathbb{N}}$ such that $\delta_{i} \rightarrow 0$ as $i \rightarrow \infty$ and

$$
d_{H}\left(\Omega_{t}^{\delta_{i}}, \Omega_{t}^{\infty}\right) \rightarrow 0
$$

for some $\left(\Omega_{t}^{\infty}\right)_{t \geq 0} \subset S_{r_{1}, R_{1}}$ locally uniformly in time as $i$ goes to infinity. As a consequence, $\left|\Omega_{t}^{\infty}\right|=1$ for all $t>0$. Here $\left(\Omega_{t}^{\delta}\right)_{t \geq 0}$ is a unique solution of (1.3) given in Proposition 3.2.

Let us briefly explain the outline of proof. Based on KK18, we first show that for a small $\delta(1.3)$ is well-posed and $\Omega_{t}^{\delta}$ is star-shaped with respect to a ball (See Definition 2.2) in Proposition 3.2. In Proposition 3.7 based on geometric properties in Lemma 3.5. we show that $\Omega_{t}^{\delta}$ is hölder continuous with respect to time. Then, by the equicontinuity of $\left(\Omega_{t}^{\delta}\right)_{t \geq 0}$ with respect to both time and space, there exists a converging subsequence. 
Proposition 3.2. Let $\delta \in\left(0, \delta_{0}\right)$ for $\delta_{0}$ given in (3.3).

(1) There exists $r_{1}=r_{1}\left(\Omega_{0}\right)$ and $R_{1}=R_{1}\left(\Omega_{0}\right)>0$ such that $\Omega_{t}^{\delta} \in S_{r_{1}, R_{1}}$ for all $t \geq 0$.

(2) There exists a unique viscosity solution $\left(\left(\Omega_{t}^{\delta}\right)_{t \geq 0}, \lambda_{\delta}\right)$ of (1.3) that is bounded and has smooth boundaries.

Proof. First, let us show that $\gamma_{\delta}$ given in (1.4) satisfies [KK18, Assumption A] for all $\delta \in\left(0, \delta_{0}\right)$. As $\gamma_{\delta}$ is a decreasing function, it holds that

$$
\gamma_{\delta}(|\Omega|) \geq \gamma_{\delta}\left(\left|B_{5 \rho}\right|\right)>\frac{n-1}{\rho} \text { for all } \Omega \subset \bar{B}_{5 \rho} \text { and all } \delta \in\left(0, \delta_{0}\right)
$$

where

$$
\delta_{0}:=\frac{\rho\left(1-\left|B_{5 \rho}\right|\right)}{n-1}
$$

Note that $1-\left|B_{5 \rho}\right|>0$ from (1.5), and thus we get $\delta_{0}>0$. On the other hand, $\gamma_{\delta}$ is Lipschitz continuous and satisfies

$$
\limsup _{R \rightarrow \infty} \frac{\gamma_{\delta}\left(\left|B_{R}\right|\right)}{R}=-\infty<\infty .
$$

Thus, we conclude that $\gamma_{\delta}$ satisfies [KK18, Assumption A] for all $\delta \in\left(0, \delta_{0}\right)$.

From Theorem 1 and 2 in KK18 this problem is well-posed and $\left(\Omega_{t}^{\delta}\right)_{t \geq 0}$ satisfies $\rho$-reflection for all $\delta \in\left(0, \delta_{0}\right)$. Furthermore, KK18, Equation (3.11)] implies that $\left(\Omega_{t}^{\delta}\right)_{t \geq 0} \subset S_{r_{1}}$ where $r_{1}=r_{1}\left(\Omega_{0}\right)$ is given by

$$
r_{1}:=\rho\left(\beta_{1}^{2}+2 \beta_{1}\right)^{\frac{1}{2}}
$$

for some $\beta_{1}>0$ such that $\bar{B}_{\left(1+\beta_{1}\right) \rho} \subset \Omega_{0}$. On the other hand due to Lemma C.2, $\Omega_{0} \subset \subset B_{R_{1}}$ and $\lambda_{\delta}(t)<0$ if $\sup _{x \in \Omega_{t}^{\delta}}|x| \geq R_{1}$, where

$$
R_{1}:=5 \rho+w_{n}^{\frac{1}{n}} \text { and } w_{n}:=\left|B_{1}(0)\right| .
$$

A barrier argument with $B_{R_{1}}$ yields that $\Omega_{t}^{\delta} \subset B_{R_{1}}$ for all $t>0$ and all $\delta \in\left(0, \delta_{0}\right)$.

The following discrete time scheme is a simplified version of Definition 5.1 in KK18.

\section{Definition 3.3.}

- The one-step discrete gradient flow with a time step $h>0, T=T(\cdot ; h, \delta) \subset \mathbb{R}^{n}$, is defined by

$$
T(E ; h, \delta) \in \underset{F \in S_{r_{0}, R_{0}}}{\arg \min } \mathcal{J}_{\delta}(F)+\frac{1}{h} \tilde{d}^{2}(F, E), \quad \mathcal{J}_{\delta}(\Omega):=\operatorname{Per}(\Omega)+\frac{1}{2 \delta}(1-|\Omega|)^{2},
$$

where pseudo-distance $\tilde{d}$ is given by

$$
\tilde{d}(F, E):=\left(\int_{E \triangle F} d(x, \partial E) d x\right)^{\frac{1}{2}}
$$

Here, $r_{0}$ and $R_{0}$ are constants such that

$$
r_{0} \in\left(0, r_{1}\right) \text { and } R_{0}>R_{1}
$$

for $r_{1}$ and $R_{1}$ given in Proposition 3.2

- The discrete gradient flow with a time step $h>0$ and the initial set $E_{0}, E_{t}=E_{t}(h, \delta) \subset \mathbb{R}^{n}$, can be defined by for $t \in \mathbb{R}_{0}^{+}$

$$
E_{t}=E_{t}(h, \delta):=T^{[t / h]}\left(E_{0} ; h, \delta\right) .
$$

Here, $T^{m}$ for $m \in \mathbb{N}$ is the $m$ th functional power.

Now, we show that $\left(\Omega_{t}^{\delta}\right)_{t \geq 0}$ can be approximated locally uniformly by above discrete flow. In Lemma B.3. we get short-time star-shapedness based on Hölder continuity of $\Omega_{t}^{\delta}$ in time. We postpone the proof into Appendix B as other arguments are parallel to [KK18, Theorem 6.8].

Proposition 3.4. Let $\delta \in\left(0, \delta_{0}\right)$ for $\delta_{0}$ given in (3.3). There exists $\left\{h_{i}\right\}_{i \in \mathbb{N}}$ such that $h_{i} \rightarrow 0$ as $i \rightarrow \infty$ and

$$
\lim _{i \rightarrow \infty} \sup _{t \in\left[t_{1}, t_{2}\right]} d_{H}\left(E_{t}\left(h_{i}, \delta\right), \Omega_{t}^{\delta}\right)=0
$$

for any $0 \leq t_{1}<t_{2}$. 
Next, we show the Hölder continuity in time in Proposition 3.7 Let us recall some results that concern sets in $S_{r, R}$ :

Lemma 3.5. [KK18, Lemma C.1] For $E_{1}, E_{2} \in S_{r, R}$ and $R>r>0$, the following holds for some $\mathcal{K}_{1}=\mathcal{K}_{1}(r, R)>0$ :

$$
d_{H}\left(E_{1}, E_{2}\right)^{n+1} \leq \mathcal{K}_{1} \widetilde{d}^{2}\left(E_{1}, E_{2}\right), \quad d_{H}\left(E_{1}, E_{2}\right)^{n+1} \leq \mathcal{K}_{1} \widetilde{d}^{2}\left(E_{1}, E_{2}\right) .
$$

Lemma 3.6. KKK18, Lemma 5.3]. For $\left(E_{t}\right)_{t>0}$ in Definition 3.3, the following holds for some $\mathcal{K}_{2}=$ $\mathcal{K}_{2}\left(r_{0}, R_{0}\right)$ and all $0<t_{1}<t_{2}$ :

$$
\widetilde{d}^{2}\left(E_{t_{2}}, E_{t_{1}}\right) \leq \mathcal{K}_{2}\left(t_{2}-t_{1}\right)\left(\mathcal{J}_{\delta}\left(E_{t_{1}}\right)-\mathcal{J}_{\delta}\left(E_{t_{2}}\right)\right)
$$

Lemma 3.5 and Lemma 3.6 imply uniform Hölder continuity in time with respect to $\delta$.

Proposition 3.7. There exists $\mathcal{K}_{3}=\mathcal{K}_{3}\left(r_{0}, R_{0}\right)$, which is independent on $\delta>0$ such that for all $0<t_{1}<t_{2}$, it holds that

$$
d_{H}\left(\Omega_{t_{1}}^{\delta}, \Omega_{t_{2}}^{\delta}\right) \leq \mathcal{K}_{3}\left(t_{2}-t_{1}\right)^{\frac{1}{n+1}} \operatorname{Per}\left(\Omega_{0}\right)^{\frac{1}{n+1}} .
$$

Proof. Note that $\mathcal{J}_{\delta}\left(E_{t}\right)$ is nonnegative and decreases in time from the construction of $E_{t}$ in Definition 3.3. Thus, Lemma 3.6 implies that

$$
\widetilde{d}^{2}\left(E_{t_{2}}, E_{t_{1}}\right) \leq \mathcal{K}_{2}\left(t_{2}-t_{1}\right)\left(\mathcal{J}_{\delta}\left(E_{t_{1}}\right)-\mathcal{J}_{\delta}\left(E_{t_{2}}\right)\right) \leq \mathcal{K}_{2}\left(t_{2}-t_{1}\right) \mathcal{J}_{\delta}\left(\Omega_{0}\right)
$$

for all $0<t_{1}<t_{2}$ and $\mathcal{K}_{2}=\mathcal{K}_{2}\left(r_{0}, R_{0}\right)$ given in Lemma 3.6. Note that $\left|\Omega_{0}\right|=1$ implies

$$
\mathcal{J}_{\delta}\left(\Omega_{0}\right)=\operatorname{Per}\left(\Omega_{0}\right)+\frac{1}{2 \delta}\left(1-\left|\Omega_{0}\right|\right)^{2}=\operatorname{Per}\left(\Omega_{0}\right)
$$

for all $\delta>0$. From Lemma 3.5 and (3.16), there exists $\mathcal{K}_{3}=\mathcal{K}_{3}\left(r_{0}, R_{0}\right)$ such that for all $0<t_{1}<t_{2}$

$$
d_{H}\left(E_{t_{2}}, E_{t_{1}}\right) \leq \mathcal{K}_{3}\left(t_{2}-t_{1}\right)^{\frac{1}{n+1}} \operatorname{Per}\left(\Omega_{0}\right)^{\frac{1}{n+1}} .
$$

As $E_{t}=E_{t}(h, \delta)$ converges to $\Omega_{t}^{\delta}$ locally uniformly as $h \rightarrow 0$ and $M \rightarrow \infty$ from Proposition 3.2 , it holds that

$$
d_{H}\left(E_{t_{2}}, E_{t_{1}}\right) \rightarrow d_{H}\left(\Omega_{t_{2}}^{\delta}, \Omega_{t_{1}}^{\delta}\right) \text { as } h \rightarrow 0, M \rightarrow \infty .
$$

Thus, from (3.17) and (3.18), we conclude (3.14)

Proof of Theorem 3.1 By Proposition 3.2 and Proposition 3.7 $\left(\Omega_{t}^{\delta}\right)_{t \geq 0}$ are equicontinuity on both space and time. Therefore, there exists a sequence $\left\{\delta_{i}\right\}_{i \in \mathbb{N}}$ such that

$$
d_{H}\left(\Omega_{t}^{\delta_{i}}, \Omega_{t}^{\infty}\right) \rightarrow 0
$$

locally uniformly in time as $i$ goes to infinity for some $\left(\Omega_{t}^{\infty}\right)_{t \geq 0} \subset S_{r_{1}, R_{1}}$. By Lemma C.5, we conclude that $\left|\Omega_{t}^{\infty}\right|=1$ for all $t>0$.

Before we finish this section, we show by example that the constraint $S_{r, R}$ on the geometry of $\Omega_{t}^{\delta}$ is needed to obtain (3.12).

Example 3.8. Consider $\left\{E_{\varepsilon}\right\}_{\varepsilon \in(0,1)}$ defined by

$$
E_{\varepsilon}:=B_{1}(0) \cup I C\left(2 e_{1}, \varepsilon\right)
$$

Here, $I C(x, r)$ is an interior cone defined in (C.1) and $e_{1}$ is a unit vector in the positive $x_{1}$ direction. Note that $r>0$ satisfying that $\left\{E_{\varepsilon}\right\}_{\varepsilon \in(0,1)} \subset S_{r}$ does not exist. It holds that $d_{H}\left(B_{1}(0), E_{\varepsilon}\right)=1$ but $\left|B_{1}(0) \triangle E_{\varepsilon}\right| \rightarrow 0$ as $\varepsilon \rightarrow 0$. As $E_{r} \subset B_{2}(0)$ for all $\varepsilon \in(0,1)$, it holds that

$$
\left.\widetilde{d}^{2}\left(B_{1}(0), E_{\varepsilon}\right)\right) \leq 4\left|B_{1}(0) \triangle E_{\varepsilon}\right| \text {. }
$$

Thus, $\left.\widetilde{d}\left(B_{1}(0), E_{\varepsilon}\right)\right)$ also converges to zero as $\varepsilon \rightarrow 0$. Therefore, $\mathcal{K}_{1}$ satisfying (3.12) for $\left\{E_{\varepsilon}\right\}_{\varepsilon \in(0,1)}$ does not exist.

On the other hand, Consider $\left\{F_{k}\right\}_{k \in \mathbb{N}}$ defined by $F_{k}:=I C\left((k+1) e_{1}, 1\right)$, which are not uniformly bounded. By the direct computation, it holds that

$$
d_{H}\left(F_{k}, B_{1}(0)\right)=k \text { and } \widetilde{d}^{2}\left(F_{k}, B_{1}(0)\right) \leq n w_{n} k^{2} .
$$

where $w_{n}$ is a volume of an unit ball in $\mathbb{R}^{n}$. Thus, we cannot find $\mathcal{K}_{1}$ such that (3.12) holds for $\left\{F_{k}\right\}_{k \in \mathbb{N}}$. 


\section{Uniform $L^{2}$ Estimates of the Lagrange Multiplier and Existence}

In this section we establish uniform $L^{2}$ estimates of $\lambda_{\delta}$ which yields the weak $L^{2}$ limit of $\lambda_{\delta}$ in Theorem 4.1. Combining with the stability of viscosity solutions in Theorem 2.12, we show the existence of solution of (1.1) in Corollary 4.2 Following the outline given in [MSS16, the estimates for our constrained discrete gradient flow defined in (3.10). Our new challenge lies in constructing local variations given in Definition 4.6 which stays in our admissible set $S_{r_{0}, R_{0}}$ (See Lemma 4.7 and Lemma 4.8).

Theorem 4.1. Let $\delta \in\left(0, \delta_{0}\right)$ for $\delta_{0}$ given in (3.3) and $\lambda_{\delta}$ be given in (1.3). There exists $\sigma_{1}=$ $\sigma_{1}\left(r_{0}, R_{0}\right)>0$ such that

$$
\left\|\lambda_{\delta}\right\|_{L^{2}([0, T])}^{2} \leq \sigma_{1}\left(\operatorname{Per}\left(\Omega_{0}\right)+T\right)
$$

Here, $r_{0}$ and $R_{0}$ are given in (3.9). As a consequence, there exists a subsequence $\left\{\delta_{i_{j}}\right\}_{j \in \mathbb{N}}$ of $\left\{\delta_{i}\right\}_{i \in \mathbb{N}}$ in Theorem 3.1 such that $\left\{\lambda_{\delta_{i_{j}}}\right\}_{j \in \mathbb{N}}$ weakly converges to $\lambda_{\infty}$ in $L^{2}([0, T])$ satisfying (4.1).

Before proving the above theorem, let us show the existence of a viscosity solution of (1.1).

Corollary 4.2. $\left(\left(\Omega_{t}^{\infty}\right)_{t \geq 0}, \lambda_{\infty}\right)$ is a viscosity solution (See Definition 1.1) of (1.1). Here, $\left(\Omega_{t}^{\infty}\right)_{t \geq 0}$ and $\lambda_{\infty}$ are given in Theorem 3.1 and Theorem 4.1. respectively.

Proof. Note that $\left(\Omega_{t}^{\delta}\right)_{t \geq 0}$ given in Proposition 3.2 is a viscosity solution of $V=-H+\lambda^{\delta}(t)$ in the sense of Definition 2.8 (See Remark 2.9). The uniform boundedness of $\left\{\lambda_{\delta}\right\}_{j \in \mathbb{N}}$ in $L^{2}$ given in Theorem 4.1]implies the equicontinuity of $\left\{\Lambda_{\delta}\right\}_{j \in \mathbb{N}}$ where $\Lambda_{\delta}(t):=\int_{0}^{t} \lambda_{\delta}(s) d s$. From Arzela-Ascoli Thereom, $\left\{\Lambda_{\delta}(t)\right\}_{k \in \mathbb{N}}$ locally uniformly converges to $\Lambda_{\infty}(t):=\int_{0}^{t} \lambda_{\infty}(s) d s$ along a subsequence. From Theorem 2.12. Theorem 3.1 and Lemma C.6 we conclude that $\left(\Omega_{t}^{\infty}\right)_{t \geq 0}$ is a viscosity solution of $V=-H+\lambda_{\infty}(t)$.

On the other hand, from Theorem 3.1, $\left|\Omega_{t}^{\infty}\right|=\left|\Omega_{0}\right|$ for all $t \geq 0$. Thus, we conclude that $\left(\left(\Omega_{t}^{\infty}\right)_{t \geq 0}, \lambda_{\infty}\right)$ is a viscosity solution of (1.1).

Let us briefly explain the outline of proof. First, in Proposition 4.5, we show that $\|d(\cdot, \partial E)\|_{L^{2}(\partial F)}$ is bounded by $\widetilde{d}(F, E)$ given in (3.8) up to a constant for any sets $E, F \in S_{r, R}$. The proof is based on the density estimates and Besicovitch's Covering Theorem.

On the other hand, we recall the discrete scheme $E_{t}=E_{t}(h, \delta)$ in (3.10) and define the corresponding Lagrange multiplier $\lambda_{\delta}^{h}(t)$ in (4.18). In Proposition 4.9, we show that the $\lambda_{\delta}^{h}(t)$ is bounded by $\frac{1}{h}\left\|d\left(\cdot, \partial E_{t-h}\right)\right\|_{L^{2}\left(\partial E_{t}\right)}$ up to a constant. By combining these two propositions with the inequality from Lemma 3.6 we conclude that $L^{2}$ norm of $\lambda_{\delta}^{h}$ is uniformly bounded. Here, we construct a local variation (See Lemma 4.7 and Lemma 4.8) in order to find the Euler-Lagrange equation.

Here is density estimates for $S_{r, R}$. We postpone the proof into the Appendix Clas the proof is classical.

Lemma 4.3. For $E \in S_{r, R}$ and $0<r<R$, the following holds: there exists $\varepsilon_{0}=\varepsilon_{0}(r, R), \eta_{i}=\eta_{i}(r, R)$ for $i=1,2$ and 3 such that for all $\varepsilon \in\left[0, \varepsilon_{0}\right]$ and $x \in \partial E$

$$
\eta_{1} \varepsilon^{n} \leq \min \left\{\left|B_{\varepsilon}(x) \backslash E\right|,\left|E \cap B_{\varepsilon}(x)\right|\right\}
$$

and

$$
\eta_{3} \varepsilon^{n-1} \leq \operatorname{Per}\left(E ; B_{\varepsilon}(x)\right) \leq \eta_{2} \varepsilon^{n-1}
$$

where

$$
\operatorname{Per}(E ; F):=\sup \left\{\int_{E} \operatorname{div} T(x) d x: T \in \mathcal{C}_{c}^{1}\left(F ; \mathbb{R}^{n}\right), \sup _{F}|T| \leq 1\right\}
$$

Note that for any $F \subset \mathbb{R}^{n}$ and $E \subset \mathbb{R}^{n}$, which has a Lipschitz boundary, it holds that

$$
P(E ; F)=\mathcal{H}^{n-1}(F \cap \partial E)
$$

(See Remark 9.5 and Example 12.6 in Mag12).

The density estimates in Lemma 4.3 and Besicovitch's Covering Theorem in Lemma 4.4 imply the following. A similar inequality was proven for the discrete gradient flow in [MSS16, Lemma 3.4.1]. We extend this results for sets in $S_{r, R}$. 
Lemma 4.4. [EG92, Theorem 1.27] [Mag12, Theorem 5.1] (Besicovitch's Covering Theorem) There exists a positive constant $\xi=\xi(n)$ with the following property: if $\mathcal{F}$ is a family of closed non-degenerate balls of $\mathbb{R}^{n}$, and the set $\mathcal{N}$ of the centers of the balls in $\mathcal{F}$ is bounded, then there exists at most countable $\mathcal{F}_{1}, \ldots, \mathcal{F}_{\xi}$ subfamilies of disjoint balls in $\mathcal{F}$ such that

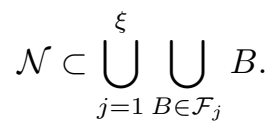

Proposition 4.5. For $E, F \in S_{r, R}$ and $0<r<R$, the following holds: for some $\sigma_{2}=\sigma_{2}(r, R)$

$$
\int_{\partial F} d^{2}(x, \partial E) d \sigma \leq \sigma_{2} \widetilde{d}^{2}(F, E)
$$

Here, $\widetilde{d}$ is given in (3.8).

Proof. 1. For all $i \in \mathbb{Z}$, define

$$
\mathcal{D}_{i}:=\left\{x \in \mathbb{R}^{n}: 2^{i}<d(x, \partial E) \leq 2^{i+1}\right\} \text { and } \delta_{0}:=\min \left\{\frac{\varepsilon_{0}}{R}, 1\right\}
$$

where $\varepsilon_{0}$ is given in Lemma 4.3. Let us show that there exists $c_{1}=c_{1}(r, R)$ such that for all $x_{i} \in \mathcal{D}_{i} \cap \partial F$

$$
\mathcal{I}_{1} \leq c_{1} \mathcal{I}_{2}, \quad \mathcal{I}_{1}:=\int_{\partial F \cap B_{2^{i-1} \delta_{0}}\left(x_{i}\right)} d^{2}(x, \partial E) d \sigma \text { and } \mathcal{I}_{2}:=\int_{(E \triangle F) \cap B_{2^{i-1} \delta_{0}}\left(x_{i}\right)} d(x, \partial E) d x .
$$

As $E, F \subset B_{R}$, it holds that for $2^{i}>2 R$,

$$
\mathcal{D}_{i} \cap \partial F=\emptyset
$$

Thus, it is enough to consider $i \leq \log _{2} R+1$. Then, it holds that

$$
2^{i-1} \delta_{0} \leq R \delta_{0} \leq \varepsilon_{0}
$$

For any $x \in B_{2^{i-1} \delta_{0}}\left(x_{i}\right)$ and $x_{i} \in \mathcal{D}_{i}$, it hold that

$$
2^{i-1} \leq d(x, \partial E) \leq 2^{i+2} .
$$

Therefore, $\mathcal{I}_{1}$ and $\mathcal{I}_{2}$ are bounded as follows;

$$
\mathcal{I}_{1} \leq \operatorname{Per}\left(F ; B_{2^{i-1} \delta_{0}}\left(x_{i}\right)\right) 2^{2 i+4} \text { and } \mathcal{I}_{2} \geq\left|(E \triangle F) \cap B_{2^{i-1} \delta_{0}}\left(x_{i}\right)\right| 2^{i-1} \delta_{0} \text {. }
$$

By (4.9) and (4.3) in Lemma 4.3, it holds that

$$
\mathcal{I}_{1} \leq \eta_{2} 2^{(i-1)(n-1)} 2^{2 i+4}=\eta_{2} 2^{i(n+1)-n+5} \delta_{0}^{n-1}
$$

On the other hand, as $B_{2^{i-1} \delta_{0}}\left(x_{i}\right) \subset E$ or $B_{2^{i-1} \delta_{0}}\left(x_{i}\right) \subset E^{c}$, it holds that

$$
\left|(E \triangle F) \cap B_{2^{i-1} \delta_{0}}\left(x_{i}\right)\right|= \begin{cases}\left|B_{2^{i-1} \delta_{0}}\left(x_{i}\right) \backslash F\right| & \text { if } B_{2^{i-1} \delta_{0}}\left(x_{i}\right) \subset E, \\ \left|B_{2^{i-1} \delta_{0}}\left(x_{i}\right) \cap F\right| & \text { if } B_{2^{i-1} \delta_{0}}\left(x_{i}\right) \subset E^{c}\end{cases}
$$

From (4.9) and (4.2) in Lemma 4.3, in both cases, we have

$$
\mathcal{I}_{2} \geq \frac{2^{(i-1)(n+1)}}{\eta_{1}}=\frac{2^{i(n+1)-n-1}}{\eta_{1}} \delta_{0}^{n} .
$$

From (4.12) and (4.14), (4.7) holds for $c_{1}:=\frac{2^{6} \eta_{1} \eta_{2}}{\delta_{0}}$.

2. Let $\mathcal{F}:=\left\{B_{2^{i-1} \delta_{0}}\left(x_{i}\right): x_{i} \in D_{i}\right\}$. Then, by Lemma 4.4, there exists $\mathcal{F}_{1}, \ldots, \mathcal{F}_{\xi}$ subfamilies of disjoint balls in $\mathcal{F}$ such that each family $\mathcal{F}_{j}$ is at most countable and

$$
\partial F \cap D_{i} \subset \bigcup_{j=1}^{\xi} \bigcup_{B \in \mathcal{F}_{j}} B
$$

From (4.15) and (4.7) in Step 1, it holds that

$$
\mathcal{I}_{3}:=\int_{\partial F \cap D_{i}} d^{2}(x, \partial E) d \sigma \leq \sum_{j=1}^{\xi} \sum_{B \in \mathcal{F}_{j}} \int_{\partial F \cap B} d^{2}(x, \partial E) d \sigma \leq c_{1} \sum_{j=1}^{\xi} \sum_{B \in \mathcal{F}_{j}} \int_{(E \triangle F) \cap B} d(x, \partial E) d x
$$


As (4.10) implies $B \subset \mathcal{D}_{i-1} \cup \mathcal{D}_{i} \cup \mathcal{D}_{i+1}$ for all $B \in \mathcal{F}$ and $\mathcal{F}_{j}$ is a family of disjoint balls, we conclude that

$$
\mathcal{I}_{3} \leq c_{1} \sum_{j=1}^{\xi} \int_{(E \triangle F) \cap\left(\mathcal{D}_{i-1} \cup \mathcal{D}_{i} \cup \mathcal{D}_{i+1}\right)} d(x, \partial E) d x=c_{1} \xi \int_{(E \triangle F) \cap\left(\mathcal{D}_{i-1} \cup \mathcal{D}_{i} \cup \mathcal{D}_{i+1}\right)} d(x, \partial E) d x
$$

3. From $\bigcup_{i \in \mathbb{Z}} \mathcal{D}_{i}=\mathbb{R}^{n}$, (4.8) and (4.17), it holds that

$\int_{\partial F} d^{2}(x, \partial E) d \sigma=\sum_{i \in \mathbb{Z}} \int_{\partial F \cap \mathcal{D}_{i}} d^{2}(x, \partial E) d \sigma \leq c_{1} \xi \sum_{i \in \mathbb{Z}} \int_{(E \triangle F) \cap\left(\mathcal{D}_{i-1} \cup \mathcal{D}_{i} \cup \mathcal{D}_{i+1}\right)} d(x, \partial E) d x=3 c_{1} \xi \widetilde{d}^{2}(F, E)$.

Thus, 4.5 holds for $\sigma_{2}:=3 c_{1} \xi$.

Now, let us find the Euler-Lagrange equation as [MSS16, Lemma 3.4.2] and Mag12, Theorem 17.20]. Consider the discrete flow $E_{t}=E_{t}(h, \delta)$ given in (3.10) and define the Lagrange multiplier at each time step.

$$
\lambda_{\delta}^{h}(t):=\gamma_{\delta}\left(\left|E_{t}(h, \delta)\right|\right)
$$

Definition 4.6. Mag12, Chapter 17.3] We say that $\left\{f_{s}\right\}_{-\varepsilon_{1}<s<\varepsilon_{2}}$ is a local variation in $A$ for an open set $A$ if for a fixed $-\varepsilon_{1}<s<\varepsilon_{2}$ and $\varepsilon_{1}, \varepsilon_{2}>0, f_{s}: \mathbb{R}^{n} \rightarrow \mathbb{R}^{n}$ is a diffeomorphism of $\mathbb{R}^{n}$ such that

$$
\begin{gathered}
f_{0}(x)=x \text { for all } x \in \mathbb{R}^{n}, \\
\left\{x \in \mathbb{R}^{n}: f_{s}(x) \neq s\right\} \subset \subset A \text { for all }-\varepsilon_{1}<s<\varepsilon_{2} .
\end{gathered}
$$

Let us denote the initial velocity of $\left\{f_{s}\right\}_{-\varepsilon_{1}<s<\varepsilon_{2}}$ by

$$
\Psi(x):=\left.\frac{\partial f_{s}}{\partial s}\right|_{s=0}(x)
$$

Recall the first variation of perimeter and volume from Theorem 17.5 and Proposition 17.8 in Mag12. For $E \in S_{r, R}$, it holds that

$$
\begin{gathered}
\operatorname{Per}\left(f_{s}(E)\right)=\operatorname{Per}(E)+s \int_{\partial E} \operatorname{div} \partial E \Psi d \mathcal{H}^{n-1}+O\left(s^{2}\right), \\
\left|f_{s}(E)\right|=|E|+s \int_{\partial E} \Psi \cdot \vec{n} d \mathcal{H}^{n-1}+O\left(s^{2}\right) .
\end{gathered}
$$

where $\operatorname{div}_{\partial E}$ is the boundary divergence on $\partial E$ defined by

$$
\operatorname{div}_{\partial E} \Psi(x):=\operatorname{div} \Psi(x)-(\vec{n} \cdot \nabla \Psi \vec{n})(x)
$$

for $x \in \partial E$. On the other hand, the first variation of $\widetilde{d}$ is as follows,

$$
\widetilde{d}^{2}\left(f_{s}(E), F\right)=\widetilde{d}^{2}(E, F)+s \int_{\partial E} d_{\text {signed }}(x, \partial F) \Psi \cdot \vec{n} d \mathcal{H}^{n-1}+O\left(s^{2}\right) .
$$

from (3.1) in MSS16].

In our case, the constraints $S_{r_{0}, R_{0}}$ gives some difficulties when we choose the local variation. The following two lemmas construct the local variations within the constraint. The first lemma discusses creating a larger perturbed set by dilation. For $a>0$, let us denote $a E:=\left\{x: a^{-1} x \in E\right\}$.

Lemma 4.7. Let $E_{t}\left(h_{i}, \delta\right)$ be given in (3.10), $\delta_{0}$ in (3.3), and $r_{0}, R_{0}$ in (3.9). Then for $0<\delta<\delta_{0}$ There exists $i^{*}=i^{*}(\delta)$ and a constant $s_{1}>0$ such that for all $i \geq i^{*}$ and $s \in\left[0, s_{1}\right)$ we have

$$
(1+s) E_{t}\left(h_{i}, \delta\right) \in S_{r_{0}, R_{0}} \text { for } t \in[0, T] .
$$

Proof. From Proposition 3.2, $B_{r_{1}}(0) \subset \Omega_{t}^{\delta} \subset B_{R_{1}}(0)$ for all $t>0$. Let us first show that there exists $i^{*}=i^{*}(\delta)$ such that for all $i \geq i^{*}, t \in[0, T]$ we have

$$
B_{r_{2}}(0) \subset E_{t}\left(h_{i}, \delta\right) \subset B_{R_{2}}(0), \quad \text { where } R_{2}:=\frac{R_{0}+R_{1}}{2} \text { and } r_{2}:=\frac{r_{0}+r_{1}}{2}
$$

By the uniform convergence of $E_{t}\left(h_{i}\right)$ in $[0, T]$ from Proposition 3.4, there exists $i^{*}=i^{*}(\delta)$ such that

$$
d_{H}\left(E_{t}\left(h_{i}\right), \Omega_{t}^{\delta}\right) \leq \min \left\{\frac{R_{0}-R_{1}}{4}, \frac{r_{0}-r_{1}}{4}\right\}
$$


for all $i \geq i^{*}$ and $t \in[0, T]$. This implies (4.27). From (4.27), we conclude that for all $s \in\left[0, s_{1}\right)$

$$
(1+s) E_{t}\left(h_{i}, \delta\right) \in B_{R_{0}} \text { where } s_{1}:=\frac{R_{0}}{R_{2}}-1
$$

As $E_{t} \in S_{r_{0}}$, C.11) imply that for all $x \in \partial E_{t}$

$$
I C(r, x) \subset E_{t} .
$$

Since $(1+s) I C(r, x)=I C((1+s) r,(1+s) x))$, we conclude that for all $x \in \partial(1+s)\left(E_{t}\right)$

$$
I C((1+s) r, x) \subset(1+s)\left(E_{t}\right)
$$

As $I C(r, x) \subset I C((1+s) r, x)$, (4.26) holds for $s \in\left[0, s_{1}\right)$.

Generating a smaller set that stays in $S_{r_{0}, R_{0}}$ turns out to be more delicate. For this we need perturbations that preserve $\partial B_{r_{0}}(0)$ and shrinks outside of $B_{r_{0}}(0)$. To stay within $S_{r_{0}, R_{0}}$ we must ensure that the angles of interior cone and exterior cone given in (C.1) and (C.2) do not decrease for the perturbed set. This is what we prove with a specific choice of the perturbation $\mathcal{G}_{s}$ below.

Lemma 4.8. Let $E_{t}\left(h_{0}, \delta\right), \delta_{0}, r_{0}, R_{0}$ and $i^{*}$ be as in the previous lemma. Let us define

$$
\psi:=\chi_{E_{t}\left(h_{i}, \delta\right)}-\chi_{E_{t}\left(h_{i}, \delta\right)^{C}} \text { and } \mathcal{G}_{s}[\psi](x):=\psi\left(\left(1+s\left(|x|^{2}-r_{0}^{2}\right)\right) x\right) .
$$

Then for $\delta \in\left(0, \delta_{0}\right)$ there exists $s_{2}>0$ such that

$$
\left\{\mathcal{G}_{s}[\psi]>0\right\} \in S_{r_{0}, R_{0}} \text { for } s \in\left[0, s_{2}\right), i \geq i^{*} \text { and } t \in[0, T] .
$$

Proof. We may assume that $E_{t}$ has a $\mathcal{C}^{1}$ boundary. Then, there is a $\mathcal{C}^{1}$ function $\phi: \mathbb{R}^{n} \rightarrow \mathbb{R}$ such that

$$
\{\phi>0\}=E_{t}, \quad\{\phi=0\}=\partial E_{t}, \quad\{\phi<0\}=\bar{E}_{t}^{C} \text {, and } D \phi \neq 0 \text { on } \partial E_{t} .
$$

First note that as $E_{t} \in S_{r_{0}, R_{0}}$ we have $B_{r_{0}} \subset\left\{\mathcal{G}_{s}[\phi]>0\right\} \subset B_{R_{0}}$. To show that $\left\{\mathcal{G}_{s}[\phi]>0\right\}$ is in $S_{r}$, from Lemma C.1 it is enough to show that

$$
D \mathcal{G}_{s}[\phi](x) \neq 0 \text { and }-\frac{D \mathcal{G}_{s}[\phi]}{\left|D \mathcal{G}_{s}[\phi]\right|}(x) \cdot x \geq r_{0} \text { for all } x \in\left\{\mathcal{G}_{s}[\phi]=0\right\} .
$$

For the rest of the proof we assume that $x \in\left\{\mathcal{G}_{s}[\phi]=0\right\}$.

Denote $P_{s}(x):=1+s\left(|x|^{2}-r_{0}^{2}\right)$ so that we can write $\mathcal{G}_{s}[\phi](x)=\phi\left(P_{s}(x) x\right)$, and thus $P_{s}(x) x \in\{\phi=0\}$ with $D \phi\left(P_{s}(x) x\right) \neq 0$. Observe that

$$
1 \leq P_{s}(x) \leq \frac{3}{2} \text { for } 0 \leq s<s_{2}:=\frac{1}{2\left(R_{0}^{2}-r_{0}^{2}\right)} .
$$

Since

$$
-D \mathcal{G}_{s}[\phi](x) \cdot x=-\left(|x|^{2} s+P_{s}(x)\right) D \phi\left(P_{s}(x) x\right) \cdot x,
$$

we have

$$
\left|D \mathcal{G}_{s}[\phi](x)\right|^{2}=P_{s}(x)^{2}\left|D \phi\left(P_{s}(x) x\right)\right|^{2}+4 s\left(|x|^{2} s+P_{s}(x)\right)\left(D \phi\left(P_{s}(x) x\right) \cdot x\right)^{2} .
$$

(4.35) yields

$$
\left|D \mathcal{G}_{s}[\phi](x)\right|^{2} \geq\left|D \phi\left(P_{s}(x) x\right)\right|^{2}>0 \text { for all } s \in\left[0, s_{2}\right),
$$

and thus the first condition of (4.34) is satisfied.

Let us now show the second condition of (4.34). As $\{\phi>0\} \in S_{r_{0}, R_{0}}$ and $P_{s}(x) x \in\{\phi=0\}$, Lemma C.1 implies

$$
-\frac{D \phi}{|D \phi|}\left(P_{s}(x) x\right) \cdot\left(P_{s}(x) x\right) \geq r_{0}
$$

From (4.36)

(4.35) and (4.39) imply that $-D \mathcal{G}_{s}[\phi](x) \cdot x$ is positive. Thus, it is enough to show that

$$
\mathcal{I}_{1}:=\left(-D \mathcal{G}_{s}[\phi](x) \cdot x\right)^{2}-r_{0}^{2}\left|D \mathcal{G}_{s}[\phi](x)\right|^{2} \geq 0 \text { for all } s \in\left[0, s_{2}\right) \text { and } x \in\left\{\mathcal{G}_{s}[\phi]=0\right\} \text {. }
$$

From (4.39) and (4.37), it holds that

$$
r_{0}^{2}\left|D \mathcal{G}_{s}[\phi](x)\right|^{2} \leq\left(P_{s}(x)^{4}+4 r_{0}^{2} s\left(|x|^{2} s+P_{s}(x)\right)\right)\left(D \phi\left(P_{s}(x) x\right) \cdot x\right)^{2} .
$$


From (4.36) and (4.41) it follows that

$$
\frac{\mathcal{I}_{1}}{\left(D \phi\left(P_{s}(x) x\right) \cdot x\right)^{2}} \geq\left(P_{s}(x)^{2}+4|x|^{2} s\left(|x|^{2} s+P_{s}(x)\right)\right)-\left(P_{s}(x)^{4}+4 r_{0}^{2} s\left(|x|^{2} s+P_{s}(x)\right)\right) .
$$

Using $s\left(|x|^{2}-r_{0}^{2}\right)=P_{s}(x)-1$ and factorizing the above, we conclude

$$
\frac{\mathcal{I}_{1}}{\left(D \phi\left(P_{s}(x) x\right) \cdot x\right)^{2}} \geq\left(P_{s}(x)-1\right)\left(-P_{s}(x)^{3}-P_{s}(x)^{2}+4 P_{s}(x)+4|x|^{2} s\right) .
$$

From (4.35), we conclude that $\mathcal{I}_{1} \geq 0$ for all $s \in\left[0, s_{2}\right)$ and $x \in\left\{\mathcal{G}_{s}[\phi]=0\right\}$.

From Lemma 4.7 and 4.8 , we get the following estimates.

Proposition 4.9. There exists $\sigma_{3}=\sigma_{3}\left(r_{0}, R_{0}\right)$ and $\sigma_{4}=\sigma_{4}\left(r_{0}, R_{0}\right)$ such that for all $t \in[0, T]$ and $i \geq i^{*}$

$$
\left|\lambda_{\delta}^{h_{i}}(t)\right|^{2} \leq \sigma_{3}+\frac{\sigma_{4}}{h^{2}} \int_{\partial E_{t}\left(h_{i}, \delta\right)} d^{2}\left(x, \partial E_{t-h_{i}}\right) d \sigma
$$

Here, $i^{*}$ is given in Lemma 4.7 and $E_{t}\left(h_{i}, \delta\right)$ is given in Proposition 3.4. Also, $r_{0}$ and $R_{0}$ are given in (3.9)

Proof. For simplicity, let $h=h_{i}$ for $i \geq i^{*}$.

1. First, show that if $f_{s}\left(E_{t}\right) \in S_{r_{0}, R_{0}}$ for all $s \in\left[0, s_{0}\right)$, then it holds that

$$
\lambda_{\delta}^{h}(t) \int_{\partial E_{t}} \vec{n} \cdot \Psi d \sigma \leq \int_{\partial E_{t}} \operatorname{div}_{\partial E_{t}} \Psi+\frac{1}{h} d_{\text {signed }}\left(x, \partial E_{t-h}\right) \vec{n} \cdot \Psi d \sigma .
$$

As $E_{t}$ is a minimizer of $\mathcal{J}_{\delta}(\cdot)+\frac{1}{h} \tilde{d}^{2}\left(\cdot, E_{t-h}\right)$ on $S_{r_{0}, R_{0}}$, (4.22), (4.23), and (4.24) imply that

$$
s \lambda_{\delta}^{h}(t) \int_{\partial E_{t}} \vec{n} \cdot \Psi d \sigma \leq s \int_{\partial E_{t}} \operatorname{div}_{\partial E_{t}} \Psi d \sigma+\frac{s}{h} \int_{\partial E_{t}} d_{\text {signed }}\left(x, \partial E_{t-h}\right) \vec{n} \cdot \Psi d \sigma+O\left(s^{2}\right)
$$

for all $s \in\left[0, s_{0}\right)$. Dividing both sides by $s>0$ and sending $s$ to zero, we conclude (4.45).

2. Let us find the upper bound of $\lambda_{\delta}^{h}(t)$. Recall $f_{s}(x):=x+s x$ in Lemma 4.7. Then, $f_{s}\left(E_{t}\right) \in S_{r_{0}, R_{0}}$ for $s \in\left[0, s_{1}\right)$ and $\Psi(x)=x$. From (4.45) in Step 1 and $r_{0} \leq \vec{n} \cdot x \leq R_{0}$ on $\partial E_{t}$, it holds that

$$
\lambda_{\delta}^{h}(t) \leq \frac{\int_{\partial E_{t}} \operatorname{div}_{\partial E_{t}} \Psi+\frac{1}{h} d_{\text {signed }}\left(x, \partial E_{t-h}\right) \vec{n} \cdot \Psi d \sigma}{\int_{\partial E_{t}} \vec{n} \cdot \Psi d \sigma} \leq \frac{n-1}{r_{0}}+\frac{R_{0}}{r_{0} \operatorname{Per}\left(E_{t}\right)} \frac{1}{h} \int_{\partial E_{t}} d_{\text {signed }}\left(x, \partial E_{t-h}\right) d \sigma
$$

3. Let us construct the lower bound. Define $g, f: \mathbb{R}^{n} \times\left[0, s_{2}\right) \rightarrow \mathbb{R}^{n}$ by

$$
g_{s}(x)=g(x, s):=\left(1+s\left(|x|^{2}-r^{2}\right)\right) x \text { and } f_{s}(x)=f(x, s):=\left(g_{s}\right)^{-1}(x)
$$

where $s_{2}$ is given in (4.35) in Lemma 4.8. As $g(f(x, s), s)=x$ and $\left.D g_{s}\right|_{s=0}=I$, it holds that

$$
\left.\frac{\partial f_{s}}{\partial s}\right|_{s=0}(x)=-\left.\frac{\partial g_{s}}{\partial s}\right|_{s=0}(x)=-\left(|x|^{2}-r^{2}\right) x
$$

From the above and (4.21), the initial velocity is

$$
\Psi(x)=-\left(|x|^{2}-r^{2}\right) x .
$$

From Lemma 4.8, $f_{s}\left(E_{t}\right) \in S_{r_{0}, R_{0}}$ for $s \in\left[0, s_{2}\right)$. By (4.45) and $\Psi \cdot \vec{n} \leq 0$ on $\partial E_{t}$, it holds that

$$
\lambda_{\delta}^{h}(t) \geq \frac{\int_{\partial E_{t}} \operatorname{div}_{\partial E_{t}} \Psi+\frac{1}{h} d_{\text {signed }}\left(x, \partial E_{t-h}\right) \vec{n} \cdot \Psi d \sigma}{\int_{\partial E_{t}} \vec{n} \cdot \Psi d \sigma}
$$

Note that from (4.27)

(4.52) $-R_{0}\left(R_{0}^{2}-r_{0}^{2}\right) \leq \Psi \cdot \vec{n} \leq-r_{0}\left(r_{2}^{2}-r_{0}^{2}\right)$ and $-(n+1)\left(R_{0}^{2}-r_{0}^{2}\right) \leq \operatorname{div}_{\partial E_{t}} \Psi \leq-(n-1)\left(r_{2}^{2}-r_{0}^{2}\right)$.

From (4.51) and (4.52), we conclude that

$$
\lambda_{\delta}^{h}(t) \geq \frac{(n-1)\left(r_{2}^{2}-r_{0}^{2}\right)}{R_{0}\left(R_{0}^{2}-r_{0}^{2}\right)}-\frac{1}{h \operatorname{Per}\left(E_{t}\right)} \int_{\partial E_{t}} d\left(x, \partial E_{t-h}\right) d \sigma
$$


4. From (4.47) and (4.53), there exists $c_{1}=c_{1}\left(r_{0}, R_{0}\right)$ and $c_{2}=c_{2}\left(r_{0}, R_{0}\right)$ such that

$$
\left|\lambda_{\delta}^{h}(t)\right| \leq c_{1}+\frac{c_{2}}{h \operatorname{Per}\left(E_{t}\right)} \int_{\partial E_{t}} d\left(x, \partial E_{t-h}\right) d \sigma .
$$

From (4.54), $(a+b)^{2} \leq 2\left(a^{2}+b^{2}\right)$ for $a, b \in \mathbb{R}$ and the Hölder's inequality, it holds that

$$
\left|\lambda_{\delta}^{h}(t)\right|^{2} \leq 2 c_{1}^{2}+\frac{2 c_{2}^{2}}{h^{2} \operatorname{Per}\left(E_{t}\right)^{2}}\left(\int_{\partial E_{t}} d\left(x, \partial E_{t-h}\right) d \sigma\right)^{2} \leq 2 c_{1}^{2}+\frac{2 c_{2}^{2}}{h^{2} \operatorname{Per}\left(E_{t}\right)} \int_{\partial E_{t}} d\left(x, \partial E_{t-h}\right)^{2} d \sigma
$$

By the isoperimetric inequality and $B_{r_{0}} \subset E_{t}$, we have $\operatorname{Per}\left(E_{t}\right)>c_{3}$ for some $c_{3}=c_{3}\left(r_{0}\right)$, we conclude that (4.44) holds for

$$
\sigma_{3}:=2 c_{1}^{2} \text { and } \sigma_{4}:=\frac{2 c_{2}^{2}}{c_{3}}
$$

\section{Proof of Theorem 4.1}

Let us show that $\left\|\lambda_{\delta}^{h}\right\|_{L^{2}([0, T])}^{2}$ is uniformly bounded for all $h=h_{i}$ for $i \geq i^{*}$ and all $\delta \in\left(0, \delta_{0}\right)$. Here, $\left\{h_{i}\right\}_{i \in \mathbb{N}}$ is given in Proposition 3.4 and $i^{*}=i^{*}(\delta)$ is given in Lemma 4.7 .

By Proposition 4.9 and Proposition 4.5, it holds that

$$
\left\|\lambda_{\delta}^{h}\right\|_{L^{2}([0, T])}^{2} \leq \sigma_{3} T+\frac{\sigma_{2} \sigma_{4}}{h^{2}} \int_{0}^{T} \widetilde{d}^{2}\left(E_{t}, E_{t-h}\right) d t \leq \sigma_{3} T+\frac{\sigma_{2} \sigma_{4}}{h} \sum_{k=1}^{\left[\frac{T}{h}\right]} \widetilde{d^{2}}\left(E_{k h}, E_{(k-1) h}\right) .
$$

Note that Lemma 3.6 implies

$$
\frac{1}{h} \sum_{k=1}^{\left[\frac{T}{h}\right]} \widetilde{d}^{2}\left(E_{k h}, E_{(k-1) h}\right) \leq \mathcal{K}_{2} \sum_{k=1}^{\left[\frac{T}{h}\right]}\left(\mathcal{J}_{\delta}\left(E_{(k-1) h}\right)-\mathcal{J}_{\delta}\left(E_{k h}\right)\right)=\mathcal{K}_{2}\left(\mathcal{J}\left(\Omega_{0}\right)-\mathcal{J}\left(E_{\left[\frac{T}{h}\right] h}\right)\right) \leq \mathcal{K}_{2} \operatorname{Per}\left(\Omega_{0}\right)
$$

Thus, 4.57) and (4.58) imply that

$$
\left\|\lambda_{\delta}^{h}\right\|_{L^{2}([0, T])}^{2} \leq \sigma_{3} T+\sigma_{2} \sigma_{4} \mathcal{K}_{2} \operatorname{Per}\left(\Omega_{0}\right)
$$

for all $h=h_{i}$ for $i \geq i^{*}$.

By the uniform continuity of $E_{t}(h, \delta)$ in Proposition 3.4, $\lambda_{\delta}^{h}$ given in (4.18) uniformly converges to $\lambda_{\delta}$ given in (1.3) along a subsequence. Thus, we conclude that (4.1) holds for

$$
\sigma_{1}:=\max \left\{\sigma_{3}, \sigma_{2} \sigma_{4} \mathcal{K}_{2}\right\} \text {. }
$$

Here, $\sigma_{2}$ is given in Proposition 4.9, $\sigma_{3}$ and $\sigma_{4}$ are given in Proposition 4.5 and $\mathcal{K}_{2}$ is given in Lemma 3.6. For $\delta_{i} \in\left(0, \delta_{0}\right)$ given in Theorem 3.1, $\lambda_{\delta_{i}}$ is uniformly bounded for all $i \in \mathbb{N}$. Thus, by Banach-Alaoglu Theorem, there exists a subsequence $\delta_{i_{j}}$ of $\delta_{i}$ in Theorem 3.1 such that $\lambda_{\delta_{i_{j}}}$ weakly converges to $\lambda_{\infty}$ in $L^{2}[0, T]$.

For the later purpose in Section [5, let us also construct $L^{2}$ estimates in $\left[t_{0}, t_{0}+T\right]$ for all $t_{0} \geq 0$.

Corollary 4.10. Let $\delta \in\left(0, \delta_{0}\right)$ for $\delta_{0}$ given in (3.3) and $\lambda_{\delta}$ be given in (1.3).

$$
\left\|\lambda_{\delta}\right\|_{L^{2}\left(\left[t_{0}, t_{0}+T\right]\right)}^{2} \leq \sigma_{1}\left(\operatorname{Per}\left(\Omega_{0}\right)+T\right)
$$

where $\sigma_{1}$ is given in (4.60).

Proof. As $\mathcal{J}_{\delta}\left(\Omega_{t}^{\delta}\right)$ given in (3.7) decreases in time, $\mathcal{J}_{\delta}\left(\Omega_{t}^{\delta}\right)$ is bounded by $\mathcal{J}_{\delta}\left(\Omega_{0}\right)=\operatorname{Per}\left(\Omega_{0}\right)$ for all $\delta>0$ and $t \geq 0$. From (4.57) and (4.58) in the proof of Theorem 4.1, we have

$$
\left\|\lambda_{\delta}^{h}\right\|_{L^{2}\left(\left[t_{0}, t_{0}+T\right]\right)}^{2} \leq \sigma_{1}\left(\operatorname{Per}\left(\Omega_{0}\right)+T\right)
$$

where $\sigma_{1}$ is given in (4.60). As the proof of Theorem 4.1, we conclude (4.61) 


\section{LARGe-Time Behavior}

In this section, we discuss the large-time behavior of $\left(\Omega_{t}^{\infty}\right)_{t \geq 0}$ given in Theorem 3.1. Here is the main theorem in this section.

Theorem 5.1. $\left(\Omega_{t}^{\infty}\right)_{t \geq 0}$ given in Theorem 3.1 uniformly converges to a ball of volume 1 , modulo translation. More precisely

$$
\inf \left\{d_{H}\left(\Omega_{t}^{\infty}, B_{r_{\infty}}(x)\right): x \in \bar{B}_{r_{1}}(0)\right\} \rightarrow 0 \text { as } t \rightarrow \infty,
$$

where $r_{1}$ is given in Proposition [3.2, $r_{\infty}:=\left(w_{n}\right)^{-\frac{1}{n}}$ and $w_{n}$ is a volume of an unit ball in $\mathbb{R}^{n}$.

Intuitively this convergence is due to the flow's formal gradient flow structure with respect to the perimeter energy. Unforunately, due to the lack of uniform regularity for $\Omega_{t}^{\delta}$ with respect to $\delta>0$, we are not able to directly show that $\Omega_{t}^{\infty}$ is the gradient flow of the perimeter energy in the space of sets with unit volume. Hence we instead utilize the gradient flow structure for the $\delta$-flow, as given in section 4 , to show this convergence.

The main estimate in the analysis is Lemma 5.3, where we bound the difference of total perimeter with respect to their differences in Hausdorff distance, in the class of star-shaped sets with their total curvature in $L^{2}$. Based on this estimate, we can proceed to show in (5.31) that the time integral of $\delta$-energy converges to the time integral of the perimeter energy. This now establishes the link between the gradient flow structure of $\delta$-flow and the limit flow, and the asymptotic convergence follows.

For $k \in \mathbb{N}$ we consider $\left(\left(U_{t}^{k}\right)_{t \geq 0}, \eta^{k}\right)$ defined by

$$
U_{t}^{k}:=\Omega_{t+k}^{\infty} \text { and } \eta^{k}(t):=\lambda_{\infty}(t+k) .
$$

Here, $\left(\Omega_{t}^{\infty}\right)_{t \geq 0}$ and $\lambda_{\infty}$ are given in Theorem 3.1 and Theorem 4.1, respectively.

Proposition 5.2. There exists a subsequence $\left\{k_{i}\right\}_{i \in \mathbb{N}}$ such that $\left\{\left(U_{t}^{k_{i}}\right)_{t \geq 0}\right\}_{i \in \mathbb{N}}$ locally uniformly converges to $\left(U_{t}^{\infty}\right)_{t>0} \subset S_{r_{1}, R_{1}}$ and $\left\{\eta^{k_{i}}\right\}_{i \in \mathbb{N}}$ weakly converges to $\eta^{\infty}$ in $L^{2}([0, T])$ for all $T>0$. As a consequence, $\left(U_{t}^{\infty}\right)_{t \geq 0}$ is a viscosity solution (See Definition 2.8) of $V=-H+\eta^{\infty}(t)$. Here, $r_{1}$ and $R_{1}$ are given in Proposition 3.2.

Proof. Theorem 3.1 and Proposition 3.7 imply that for all $0<k_{1}<k_{2}$

$$
d_{H}\left(U_{t}^{k_{1}}, U_{t}^{k_{2}}\right) \leq \mathcal{K}_{3}\left(k_{2}-k_{1}\right)^{\frac{1}{n+1}} \operatorname{Per}\left(\Omega_{0}\right)^{\frac{1}{n+1}} .
$$

where $\mathcal{K}_{3}$ is given in Proposition 3.7. Also, as $\left\{U_{t}^{k}\right\}_{k \in \mathbb{N}} \subset S_{r_{1}, R_{1}}$ from Theorem 3.1, we have the equicontinuity in both space and time of $\left\{U_{t}^{k}\right\}_{k \in \mathbb{N}}$.

By the equicontinuity of $\left\{U_{t}^{k}\right\}_{k \in \mathbb{N}}$ and the uniform $L^{2}$ estimates in Corollary 4.10 there exists a subsequence $\left\{k_{i}\right\}_{i \in \mathbb{N}}$ such that $\left\{\left(U_{t}^{k_{i}}\right)_{t>0}\right\}_{i \in \mathbb{N}}$ locally uniformly converges to $\left(U_{t}^{\infty}\right)_{t>0} \subset S_{r_{1}, R_{1}}$ and $\left\{\eta^{k_{i}}\right\}_{i \in \mathbb{N}}$ weakly converges to $\eta^{\infty}$ in $L^{2}([0, T])$ for all $T>0$.

Note that $\left(U_{t}^{k}\right)_{t \geq 0}$ is a viscosity solution of $V=-H+\eta^{k}(t)$. From Theorem 2.12 and Lemma C.6. $\left(U_{t}^{\infty}\right)_{t \geq 0}$ is a viscosity solution of $V=-H+\eta^{\infty}(t)$.

Now, in Lemma 5.3. we estimates the time integral of the perimeter difference for two evolving sets $\left(\Omega_{t}^{j}\right)_{t \geq 0} \subset S_{r, R}$ and $j \in\{1,2\}$.

Lemma 5.3. For $j \in\{1,2\}$, consider $\left(\Omega_{t}^{j}\right)_{t>0} \subset S_{r, R}$ for $R>r>0$ such that $\left(\partial \Omega_{t}^{j}\right)_{t>0}$ are smooth. Suppose that there exists a constant $\mathcal{W}<+\infty$ such that for $T>0$ and $j \in\{1,2\}$

$$
\int_{0}^{T} \int_{\partial \Omega_{t}^{j}} H(x, t)^{2} d \sigma d t<\mathcal{W}
$$

where $H(x, t)$ is the mean curvature at $x \in \partial \Omega_{t}^{j}$. Then, there exists a constant $\bar{m}=\bar{m}(r, R, T, \mathcal{W})>0$ such that

$$
\left(\int_{0}^{T} \operatorname{Per}\left(\Omega_{t}^{1}\right)-\operatorname{Per}\left(\Omega_{t}^{2}\right) d t\right)_{18}^{2} \leq \bar{m} \sup _{t \in[0, T]} d_{H}\left(\Omega_{t}^{1}, \Omega_{t}^{2}\right) .
$$


Proof. As $\left\{\Omega_{t}^{j}\right\}_{j \in\{1,2\}} \subset S_{r, R}$ are smooth for $t>0$, there exist two smooth functions $u_{1}, u_{2}: B_{r}^{n-1}(0) \times$ $[0, T] \rightarrow \mathbb{R}$ such that for $j=1,2$

$$
\partial \Omega_{t}^{j} \cap C_{r, R}^{+}(0)=\left\{\left(u_{j}\left(y^{\prime}, t\right), y^{\prime}\right): y^{\prime} \in B_{r}^{n-1}(0)\right\}
$$

Furthermore, from $\Omega_{1}, \Omega_{2} \in S_{r, R}$ again, there exists a constant $c_{1}=c_{1}(r, R)$ such that

(5.7) $\left\|u_{1}-u_{2}\right\|_{L^{\infty}\left(B_{r}^{n-1}(0) \times[0, T]\right)} \leq c_{1} \sup _{t \in[0, T]} d_{H}\left(\Omega_{t}^{1}, \Omega_{t}^{2}\right)$ and $\left\|\nabla u_{j}\right\|_{L^{\infty}\left(B_{r}^{n-1}(0) \times[0, T]\right)} \leq c_{1}$ for $j=1,2$.

1. Let us first show that there exists $\bar{m}_{1}=\bar{m}_{1}(r, R, T, \mathcal{W})$ for $\mathcal{W}$ given in (5.4)

$$
\left\|\vec{n}_{1}-\vec{n}_{2}\right\|_{L^{2}\left(B_{r}^{n-1}(0) \times[0, T]\right)}^{2} \leq \bar{m}_{1} \sup _{t \in[0, T]} d_{H}\left(\Omega_{t}^{1}, \Omega_{t}^{2}\right) \text { where } \vec{n}_{j}:=\frac{\left(1, \nabla u_{j}\right)}{\sqrt{1+\left|\nabla u_{j}\right|^{2}}} \text { for } j=1,2 .
$$

As $\vec{n}_{1}$ and $\vec{n}_{2}$ are unit vectors, we get the following by the direct computation,

$$
\begin{aligned}
\left|\vec{n}_{1}-\vec{n}_{2}\right|^{2} & =2\left(1-\vec{n}_{1} \cdot \vec{n}_{2}\right), \\
& \leq\left(\sqrt{1+\left|\nabla u_{1}\right|^{2}}+\sqrt{1+\left|\nabla u_{2}\right|^{2}}\right)\left(1-\vec{n}_{1} \cdot \vec{n}_{2}\right), \\
& =\left(\left(\sqrt{1+\left|\nabla u_{1}\right|^{2}}\right) \vec{n}_{1}-\left(\sqrt{1+\left|\nabla u_{2}\right|^{2}}\right) \vec{n}_{2}\right) \cdot\left(\vec{n}_{1}-\vec{n}_{2}\right)=\nabla\left(u_{1}-u_{2}\right) \cdot\left(\vec{n}_{1}^{\prime}-\vec{n}_{2}^{\prime}\right) .
\end{aligned}
$$

where $\vec{n}_{j}^{\prime}$ is the last $n-1$ components of $\vec{n}_{j}$ given by

$$
\vec{n}_{j}^{\prime}:=\frac{\nabla u_{j}}{\sqrt{1+\left|\nabla u_{j}(x)\right|^{2}}} \text { for } j \in\{1,2\} .
$$

Note that the mean curvature at $\left(u_{j}(x, t), x\right) \in \partial \Omega_{t}^{j}$ for $x \in B_{r}^{n-1}(0)$ is given by

$$
H\left(\left(u_{j}(x, t), x\right), t\right)=\nabla \cdot \vec{n}_{j}^{\prime}(x, t) .
$$

From (5.4), there exists $c_{2}=c_{2}(r, R, \mathcal{W})$ such that for $j \in\{1,2\}$

$$
\left\|\nabla \cdot \vec{n}_{j}^{\prime}\right\|_{L^{2}\left(B_{r}^{n-1}(0) \times[0, T]\right)} \leq c_{2}
$$

From integration by parts, we have

$$
\begin{aligned}
\mathcal{I}_{1} & :=\int_{B_{r}^{n-1}(0) \times[0, T]} \nabla\left(u_{1}-u_{2}\right) \cdot\left(\vec{n}_{1}^{\prime}-\vec{n}_{2}^{\prime}\right) d x d t \\
& =\int_{\partial B_{r}^{n-1}(0) \times[0, T]}\left(u_{1}-u_{2}\right)\left(\vec{n}_{1}^{\prime}-\vec{n}_{2}^{\prime}\right) \cdot \nu d \sigma d t-\int_{B_{r}^{n-1}(0) \times[0, T]}\left(u_{1}-u_{2}\right) \cdot\left(\nabla \cdot\left(\vec{n}_{1}^{\prime}-\vec{n}_{2}^{\prime}\right)\right) d x d t
\end{aligned}
$$

where $\nu$ is the outward normal vector on $\partial B_{r}^{n-1}(0)$. By applying the Hölder inequality at each terms and using (5.7) and (5.14), we have

$$
\begin{aligned}
\left|\mathcal{I}_{1}\right| & \leq\left(2 \operatorname{Per}\left(B_{r}^{n-1}\right) T+\left\|\nabla \cdot\left(\vec{n}_{1}^{\prime}-\vec{n}_{2}^{\prime}\right)\right\|_{L^{2}\left(B_{r}^{n-1}(0) \times[0, T]\right)}\left|B_{r}^{n-1}\right|^{\frac{1}{2}} T^{\frac{1}{2}}\right)\left\|u_{1}-u_{2}\right\|_{L^{\infty}\left(B_{r}^{n-1}(0) \times[0, T]\right)} \\
& \leq\left(2 \operatorname{Per}\left(B_{r}^{n-1}\right) T+2 c_{2}\left|B_{r}^{n-1}\right|^{\frac{1}{2}} T^{\frac{1}{2}}\right) c_{1} \sup _{t \in[0, T]} d_{H}\left(\Omega_{t}^{1}, \Omega_{t}^{2}\right)
\end{aligned}
$$

From (5.11) and (5.18), we conclude (5.8) with

$$
\bar{m}_{1}:=2 c_{1}\left(\operatorname{Per}\left(B_{r}^{n-1}\right) T+c_{2}\left|B_{r}^{n-1}\right|^{\frac{1}{2}} T^{\frac{1}{2}}\right)
$$

2. Let us show that there exists $\bar{m}_{2}=\bar{m}_{2}(r, R, T, \mathcal{W})$ for $\mathcal{W}$ given in (5.4)

$$
\left(\mathcal{I}_{2}\right)^{2} \leq \bar{m}_{2} \sup _{t \in[0, T]} d_{H}\left(\Omega_{t}^{1}, \Omega_{t}^{2}\right) \text { where } \mathcal{I}_{2}:=\int_{0}^{T} \operatorname{Per}\left(\Omega_{1} ; C_{r, R}^{+}(0)\right)-\operatorname{Per}\left(\Omega_{2} ; C_{r, R}^{+}(0)\right) d t
$$

Recall from (4.4) and Theorem 9.1 in Mag12, we have

$$
\int_{0}^{T} \operatorname{Per}\left(\Omega_{t}^{j} ; C_{r, R}^{+}(0)\right) d t=\int_{B_{r}^{n-1}(0) \times[0, T]} \sqrt{1+\left|\nabla u_{j}\right|^{2}} d x d t=\int_{B_{r}^{n-1}(0) \times[0, T]}\left(1, \nabla u_{j}\right) \cdot \vec{n}_{j} d x d t
$$

where $\left\{u_{j}\right\}_{j \in\{1,2\}}$ and $\left\{n_{j}\right\}_{j \in\{1,2\}}$ are given in (5.6) and (5.8), respectively. By adding and subtracting the same term in $\mathcal{I}_{2}$, we have the identity

$$
\mathcal{I}_{2}=\mathcal{I}_{3}+\mathcal{I}_{4}
$$


where

$$
\mathcal{I}_{3}:=\int_{B_{r}^{n-1}(0) \times[0, T]}\left(1, \nabla u_{1}\right) \cdot\left(\vec{n}_{1}-\vec{n}_{2}\right) d x d t
$$

and

$$
\mathcal{I}_{4}:=\int_{B_{r}^{n-1}(0) \times[0, T]}\left(\left(1, \nabla u_{1}\right)-\left(1, \nabla u_{2}\right)\right) \cdot \vec{n}_{2} d x d t=\int_{B_{r}^{n-1}(0) \times[0, T]} \nabla\left(u_{1}-u_{2}\right) \cdot \vec{n}_{2}^{\prime} d x d t
$$

Here, $\left\{n_{j}\right\}_{j \in\{1,2\}}$ and $\left\{n_{j}^{\prime}\right\}_{j \in\{1,2\}}$ are given in (5.8) and (5.12), respectively.

By applying (5.7) and (5.8) and the Hölder inequality, we get

$$
\mathcal{I}_{3}^{2} \leq\left(1+c_{1}^{2}\right)\left|B_{r}^{n-1}\right| T \bar{m}_{1} \sup _{t \in[0, T]} d_{H}\left(\Omega_{t}^{1}, \Omega_{t}^{2}\right) .
$$

where $c_{1}$ and $\bar{m}_{1}$ are given in (5.7) and (5.19). On the other hand, by the similar arguments in (5.16)

$$
\mathcal{I}_{4} \leq \bar{m}_{1} \sup _{t \in[0, T]} d_{H}\left(\Omega_{t}^{1}, \Omega_{t}^{2}\right)
$$

where $\bar{m}_{1}$ is given in (5.19). As $\left(\Omega_{t}^{j}\right)_{t \geq 0} \subset S_{r, R}$ for $j \in\{1,2\}$, we have

$$
\sup _{t \in[0, T]} d_{H}\left(\Omega_{t}^{1}, \Omega_{t}^{2}\right) \leq 2 R \text {. }
$$

Thus, (5.26) and (5.27) imply that

$$
\mathcal{I}_{4}^{2} \leq 2 \bar{m}_{1}^{2} R d_{H}\left(\Omega_{t}^{1}, \Omega_{t}^{2}\right) .
$$

From (5.22) combining with (5.25) and (5.28), we have

$$
\mathcal{I}_{2}^{2} \leq 2\left(\mathcal{I}_{3}^{2}+\mathcal{I}_{4}^{2}\right) \leq 2\left(\left(1+c_{1}^{2}\right)\left|B_{r}^{n-1}\right| T \bar{m}_{1}+2 \bar{m}_{1}^{2} R\right) \sup _{t \in[0, T]} d_{H}\left(\Omega_{t}^{1}, \Omega_{t}^{2}\right)
$$

Thus, we conclude (5.20) for

$$
\bar{m}_{2}:=2 \bar{m}_{1}\left(\left(1+c_{1}^{2}\right)\left|B_{r}^{n-1}\right| T \bar{m}_{1}+2 \bar{m}_{1}^{2} R\right) .
$$

Here, $c_{1}$ and $\bar{m}_{1}$ are given in (5.7) and (5.19).

3. As every sets in $S_{r, R}$ can be covered by a finite number of cylinders $C_{r, R}^{+}(0)$ after some rotations, (5.20) implies (5.5).

From the estimates in Lemma 5.3 and our approximation from $\left(\Omega_{t}^{\delta}\right)_{t \geq 0}$ in Theorem 3.1 we conclude that the limit flow $\left(U_{t}^{\infty}\right)_{t \geq 0}$ is stationary.

Proposition 5.4. $\left(U_{t}^{\infty}\right)_{t \geq 0}$ given in Proposition 5.2 is stationary.

Proof. 1. Let us show that there exists $\mathcal{E}^{\infty}: \mathbb{R}^{+} \rightarrow \mathbb{R}^{+}$such that

$$
\mathcal{E}^{\infty}(k):=\lim _{\delta \rightarrow 0} \mathcal{E}^{\delta}(k) \text { where } \mathcal{E}^{\delta}(k):=\int_{0}^{T} \mathcal{J}_{\delta}\left(\Omega_{t+k}^{\delta}\right) d t
$$

It is enough to show that $\left\{\mathcal{E}^{\delta}(k)\right\}_{\delta>0}$ is a Cauchy sequence as $\delta \rightarrow 0$ for all $k \in \mathbb{R}_{0}^{+}$. As $\Omega_{t}^{\delta}$ is smooth for $t>0$ from Proposition 3.2 and $\Omega_{t}^{\delta}$ is a gradient flow of $\mathcal{J}_{\delta}$, we have

$$
\int_{t_{0}}^{t_{0}+T} \int_{\partial \Omega_{t}^{\delta}} V^{2} d \sigma d t=\mathcal{J}_{\delta}\left(\Omega_{t_{0}}^{\delta}\right)-\mathcal{J}_{\delta}\left(\Omega_{t_{0}+T}^{\delta}\right) \leq \operatorname{Per}\left(\Omega_{0}\right)
$$

where $V$ is the normal velocity at $x \in \partial \Omega_{t}^{\delta}$. As $H=\lambda-V$, Corollary 4.10 and (5.32) implies the uniform bound on $\|H\|_{L^{2}\left(t_{0}, t_{0}+T ; L^{2}\left(\partial \Omega_{t}^{\delta}\right)\right)}$.

As $\mathcal{J}_{\delta}\left(\Omega_{t}^{\delta}\right)=\operatorname{Per}\left(\Omega_{t}^{\delta}\right)+2 \delta \lambda_{\delta}(t)^{2}$, Lemma 5.3 and Corollary 4.10 imply that for $\delta_{1}>\delta_{2}>0$

$$
\begin{aligned}
\left|\mathcal{E}^{\delta_{1}}(k)-\mathcal{E}^{\delta_{2}}(k)\right| & \leq\left|\int_{0}^{T} \operatorname{Per}\left(\Omega_{t+k}^{\delta_{1}}\right)-\operatorname{Per}\left(\Omega_{t+k}^{\delta_{2}}\right) d t\right|+2 \delta_{1}\left\|\lambda_{\delta_{1}}\right\|_{L^{2}([k, k+T])}^{2}+2 \delta_{2}\left\|\lambda_{\delta_{2}}\right\|_{L^{2}([k, k+T])}^{2}, \\
& \leq c\left(\sup _{t \in[0, T]} d_{H}\left(\Omega_{t}^{\delta_{1}}, \Omega_{t}^{\delta_{2}}\right)^{\frac{1}{2}}+\delta_{1}+\delta_{2}\right)
\end{aligned}
$$

where a constant $c$ is given by

$$
c:=\max \left\{\bar{m}^{\frac{1}{2}}, 2 \sigma_{1}\left(\operatorname{Per}\left(\Omega_{0}\right)+T\right)\right\} .
$$


Here, $\sigma_{1}$ and $\bar{m}$ are given in (4.60) and (5.5), respectively. From Theorem 3.1, we conclude (5.31).

2. Lemma 3.6 and the smoothness of $\Omega_{t}^{\delta}$ for $t>0$ from Proposition 3.2 imply that for $s, k \in \mathbb{R}_{0}^{+}$

$$
\int_{0}^{T} \widetilde{d}^{2}\left(\Omega_{t+k+s}^{\delta}, \Omega_{t+k}^{\delta}\right) d t \leq s \mathcal{K}_{2}\left(\mathcal{E}^{\delta}(k)-\mathcal{E}^{\delta}(k+s)\right)
$$

where $\mathcal{K}_{2}$ is given in Lemma 3.6. Taking $\delta$ into zero, (5.31) and Theorem 3.1 imply that for $s, k \in \mathbb{R}_{0}^{+}$

$$
\int_{0}^{T} \widetilde{d}^{2}\left(U_{t+s}^{k}, U_{t}^{k}\right) d t \leq s \mathcal{K}_{2}\left(\mathcal{E}^{\infty}(k)-\mathcal{E}^{\infty}(k+s)\right)
$$

where $U_{t}^{k}$ is given in (15.2).

Note that as $\mathcal{E}^{\delta}(k)$ is monotone decreasing for all $\delta>0, \mathcal{E}^{\infty}(k)$ is also monotone decreasing in $k$. Taking $k$ into $\infty$, we get for $s \in \mathbb{R}_{0}^{+}$

$$
\int_{0}^{T} \widetilde{d}^{2}\left(U_{t+s}^{\infty}, U_{t}^{\infty}\right) d t \leq s \mathcal{K}_{2}\left(\inf _{k>0} \mathcal{E}^{\infty}(k)-\inf _{k>s} \mathcal{E}^{\infty}(k)\right)=0
$$

and we conclude.

\section{Proof of Theorem 5.1}

1. Let $\eta^{\infty}$ and $U_{t}^{\infty}$ be as given in Proposition 5.2. We denote $U_{t}^{\infty}$ by $U^{\infty}$ since we know that it is stationary from the last proposition. We will show that $\eta^{\infty}$ is independent of time as well. Let us argue by contradiction, and suppose $\eta^{\infty}\left(t_{1}\right) \neq \eta^{\infty}\left(t_{2}\right)$ for two Lebesgue points $t_{1}<t_{2}$ in $\mathbb{R}_{0}^{+}$. We may assume that $\eta^{\infty}\left(t_{1}\right)<\eta^{\infty}\left(t_{2}\right)$. As $t_{1}$ and $t_{2}$ are Lebesgue points of $\eta^{\infty}$, there exists $\delta_{1}>0$ such that for any $\delta \in\left(0, \delta_{1}\right)$, we have

$$
\frac{\Upsilon\left(t_{1}+\delta\right)-\Upsilon\left(t_{1}\right)}{\delta} \leq \frac{\eta^{\infty}\left(t_{1}\right)+\eta^{\infty}\left(t_{2}\right)}{2} \leq \frac{\Upsilon\left(t_{2}+\delta\right)-\Upsilon\left(t_{2}\right)}{\delta} \text { where } \Upsilon(t):=\int_{0}^{t} \eta^{\infty}(s) d s .
$$

Therefore, for $\delta \in\left(0, \delta_{1}\right)$, we have

$$
\Upsilon\left(t_{1}+\delta\right)<\Theta_{1}\left(t_{1}+\delta\right) \text { and } \Upsilon\left(t_{2}+\delta\right)>\Theta_{2}\left(t_{2}+\delta\right)
$$

where

$$
\Theta_{i}(t):=\frac{1}{2}\left(\eta^{\infty}\left(t_{1}\right)+\eta^{\infty}\left(t_{2}\right)\right)\left(t-t_{i}\right)+\Upsilon\left(t_{i}\right) \text { for } i \in\{1,2\}
$$

From Proposition 5.2 $u(x):=\chi_{U^{\infty}}(x)-\chi_{\left(U^{\infty}\right)^{C}}(x)$ is a viscosity solution of $V=-H+\eta^{\infty}(t)$. Let us define $v_{i}: \mathbb{R}^{n} \times\left[0, \delta_{1}\right] \rightarrow \mathbb{R}$ by

$$
v_{1}(x, t):=\widetilde{u}\left(x ;\left(-\Upsilon+\Theta_{1}\right)\left(t+t_{1}\right)\right) \text { and } v_{2}(x, t):=\widehat{u}\left(x ;\left(\Upsilon-\Theta_{2}\right)\left(t+t_{2}\right)\right)
$$

Observe that $v_{1}=v_{2}=u$ at $t=0$ by definition of $\Upsilon$ and $\Theta_{i}$. Moreover by (5.39) $v_{1}$ and $v_{2}$ are each a viscosity subsolution and supersolution of $V=-H+\frac{1}{2}\left(\eta^{\infty}\left(t_{1}\right)+\eta^{\infty}\left(t_{2}\right)\right)$. Hence Theorem 2.10 implies that $v_{1} \geq v_{2}$ in $\left[0, \delta_{1}\right]$. (5.39) and $v_{1}(\cdot, \delta) \geq v_{2}(\cdot, \delta)$ can only both hold if $U^{\infty}$ is the whole $\mathbb{R}^{n}$, which is not the case here, so we reach a contradiction.

2. As $\left(U_{t}^{\infty}\right)_{t \geq 0}$ and $\eta^{\infty}$ are stationary from Proposition 5.4 and Step 1, we conclude that $U_{t}^{\infty}$ is a viscosity solution of the elliptic problem,

$$
H=\eta^{\infty}
$$

As $\left(U_{t}^{\infty}\right)_{t \geq 0} \subset S_{r_{1}, R_{1}}$ from Proposition 5.2, $\left(U_{t}^{\infty}\right)_{t \geq 0}$ can be locally represented by graphs. Then, the regularity of (5.41) in GT15, Corollary 10.7] implies that $U_{t_{1}}^{\infty}$ is smooth. As $\left(U_{t}^{\infty}\right)_{t \geq 0} \subset S_{r_{1}, R_{1}}$, we conclude that $U_{t}^{\infty}=B_{r_{\infty}}(x)$ in $\mathbb{R}_{0}^{+}$for some $x \in \bar{B}_{r_{1}}(0)$ where $r_{\infty}$ given in Theorem 5.1. Therefore, every sequence of $\left(\Omega_{t}^{\infty}\right)_{t \geq 0}$ has a subsequence converging to $B_{r_{\infty}}(x)$ for some $x \in \bar{B}_{r_{1}}(0)$, we conclude (5.1). 


\section{Appendix A. Examples of Unbounded Total Mean Curvature}

In this section, we present examples of unbounded total mean curvature for $\mathbb{R}^{2}$ in Example A.1 and $\mathbb{R}^{n}, n \geq 3$ in Example A.2. Here is an example of a nonconvex domain in $\mathbb{R}^{2}$ such that the total mean curvature is unbounded although perimeter and volume are bounded.

Example A.1. Let $\left\{O_{i}\right\}_{i \in \mathbb{N}}$ be a sequence of mutually disjoint balls in $\mathbb{R}^{2}$ with radius $r_{i}:=\frac{1}{i^{2}}$ such that $O_{i} \subset \subset B_{10}$ for all $i \in \mathbb{N}$. Define a domain $\Omega$ by

$$
\Omega:=B_{10}-\bigcup_{i=1}^{\infty} O_{i}
$$

Gauss-Bonnet Theorem implies $\int_{\partial O_{i}} H=-2 \pi$ for all $i \in \mathbb{N}$. Thus, total mean curvature is unbounded although perimeter and volume are positive and bounded as follows,

$$
|\Omega|=w_{n}\left(10^{2}-\sum_{i=1}^{\infty} \frac{1}{i^{4}}\right), \quad \operatorname{Per}(\Omega)=n w_{n}\left(10-\sum_{i=1}^{\infty} \frac{1}{i^{2}}\right)
$$

Based on Example A.1, we can construct a simply connected and star-shaped set, whose total mean curvature is unbounded for $n \geq 3$ (See example A.2 and Lemma A.3).

Example A.2. Let $\phi: \mathbb{R}^{n} \rightarrow \mathbb{R}^{+}$be a smooth function such that $\phi$ is concave,

$$
0<\phi<1 \text { in } B_{1}^{n-1}, \quad \phi=0 \text { in }\left(B_{1}^{n-1}\right)^{c} \text { and }|D \phi| \leq 1 \text { in } \mathbb{R}^{n} .
$$

Denote

(A.4) $\mathcal{D}:=\left\{\left[0, \phi\left(x^{\prime}\right)\right] \times x^{\prime}: x^{\prime} \in B_{1}^{n-1}\right\} \subset \mathbb{R}^{n}, \quad \partial^{-} D:=B_{1}^{n-1}$ and $\partial^{+} D:=\left\{\left(\phi\left(x^{\prime}\right), x^{\prime}\right): x^{\prime} \in B_{1}^{n-1}\right\}$

Here, $B_{1}^{n-1}$ is a ball of radius 1 and center 0 in $\mathbb{R}^{n-1}$.

As Example A.1 choose $\left\{x_{i}\right\}_{i \in \mathbb{N}} \subset \mathbb{R}^{n-1}$ such that $B_{r_{i}}^{n-1}\left(x_{i}\right)$ are a sequence of disjoint balls such that

$$
r_{i}:=\frac{1}{i^{\frac{1}{n-2}}} \text { and } x_{i} \in B_{10}^{n-1}
$$

Define a domain $\Omega \subset \mathbb{R}^{n}$ by

$$
\Omega:=\Omega_{1} \cup \Omega_{2} \text { where } \Omega_{1}:=[-40,40]^{n} \quad \text { and } \quad \Omega_{2}:=\bigcup_{i=1}^{\infty}\left(r_{i} \mathcal{D}+\left(40, x_{i}\right)\right) .
$$

where $e_{1}$ is a unit vector of the first axis. From Lemma A.3 $\Omega$ is star-shaped with respect to some ball.

The boundary is divided by two parts,

$$
\partial \Omega_{t}=\Gamma_{1} \cup \Gamma_{2} \text { where } \Gamma_{1}:=\left(\partial \Omega_{1} \backslash\left(\bigcup_{i=1}^{\infty} r_{i} \partial \mathcal{D}^{-}+\left(40, x_{i}\right)\right)\right) \text { and } \Gamma_{2}:=\bigcup_{i=1}^{\infty}\left(r_{i} \partial \mathcal{D}^{+}+\left(40, x_{i}\right)\right)
$$

Note that $\Gamma_{1}$ has bounded total mean curvature, but total mean curvature of $\Gamma_{2}$ is unbounded as follows. From (A.5) and the change of variables, it holds that

$$
\int_{\Gamma_{2}} H d \sigma=\sum_{i=1}^{\infty} \int_{r_{i} \partial \mathcal{D}^{+}+x_{i}} H d \sigma,=\left(\int_{\partial \mathcal{D}^{+}} H d \sigma\right) \sum_{i=1}^{\infty} r_{i}^{n-2} .
$$

Then, (A.7) implies that

$$
\int_{\Gamma_{2}} H d \sigma=\left(\int_{\partial \mathcal{D}^{+}} H d \sigma\right) \sum_{i=1}^{\infty} \frac{1}{i}
$$

while volume and perimeter are bounded as follows.

$$
|\Omega|=\left(80^{n}+|\mathcal{D}| \sum_{i=1}^{\infty} r_{i}^{n}\right), \quad \operatorname{Per}(\Omega)=\left(80^{n-1} n-\sum_{i=1}^{\infty} w_{n-1} r_{i}^{n-1}+\left|\partial^{+} \mathcal{D}\right| \sum_{i=1}^{\infty} r_{i}^{n-1}\right)
$$


Lemma A.3. There exists $r>0$ such that $\Omega$ given in Example $A .2$ is star-shaped with respect to $B_{r}$.

Proof. As $\Omega_{1}$ given in (A.6) is star-shaped, it is enough to consider a point in $\Omega_{2}$. Note that $\Gamma_{2}$ given in (A.7) is smooth. From Lemma C.1, it is enough to show that

$$
x \cdot \vec{n}_{x} \geq r
$$

for some $r>0$ and all $x \in \Gamma_{2}$. Denote $x=\left(x^{1}, x^{\prime}\right) \in \mathbb{R}^{n}$. For $x \in r_{i} \partial \mathcal{D}^{+}+\left(40, x_{i}\right)$, there exists $y_{i} \in B_{1}^{n-1}$ such that

$$
x=\left(r_{i} \phi\left(y_{i}\right), r_{i} y_{i}\right)+\left(40, x_{i}\right)
$$

and thus

$$
\vec{n}_{x}=\frac{\left(1,-D \phi\left(y_{i}\right)\right)}{\sqrt{1+\left|D \phi\left(y_{i}\right)\right|^{2}}}
$$

From (A.3) and (A.5), it holds that

$$
x \cdot \geq \frac{40}{\sqrt{1+\left|D \phi\left(y_{i}\right)\right|^{2}}}-\frac{\left|D \phi\left(y_{i}\right)\right|\left(\left|r_{i} y_{i}\right|+\left|x_{i}\right|\right)}{\sqrt{1+\left|D \phi\left(y_{i}\right)\right|^{2}}} \geq 10
$$

\section{Appendix B. Proof of Proposition 3.4}

In this section, we prove Proposition 3.4. First, in Lemma B.3 we show short-time star-shapedness based on the Hölder continuity in Lemma B.1 The remaining arguments are parallel to Theorem 6.5 in KK18. For simplicity, we fix $r_{0}$ and $R_{0}$ given in (3.9) and $\delta \in\left(0, \delta_{0}\right)$ for $\delta_{0}$ given in (3.3). Also, let $\Omega_{t}$ be a viscosity solution of $V=-H+\gamma_{\delta}\left(\left|\Xi_{t}\right|\right)$ where $\Xi_{t}$ is an energy solution given in Definition B.4.

First, let us recall several properties of solutions from [KK18.

Lemma B.1. [KK18, Corollary 2.10] Assume that $\Omega_{0} \in S_{r, R}$. Then, there exists

$$
\mathcal{M}_{1}=\mathcal{M}_{1}\left(r, R,\left\|\gamma_{\delta}\left(\left|\Xi_{t}\right|\right)\right\|_{L^{\infty}([0, T])}\right)
$$

such that we have

$$
\sup _{x \in \partial \Omega_{t}} d\left(x, \partial \Omega_{0}\right) \leq \mathcal{M}_{1}|t|^{\frac{1}{2}} \text { for } t \in[0, T] .
$$

Theorem B.2. [KK18, Theorem 3.6] Let $I=\left[0, t_{0}\right)$ be the maximal interval satisfying $\overline{B_{\rho}} \subset \Omega_{t}$. Then, $\Omega_{t}$ satisfies $\rho$-reflection in $I$.

Lemma B.1 and Theorem B.2 imply the following lemma.

Lemma B.3. (Short-time star-shapedness) For $r>r_{0}>0$ and $0<R<R_{0}$, suppose that $\bar{B}_{(1+\beta) \rho} \subset \Omega_{0}$ and $\Omega_{0} \in S_{r, R}$ for $r=\rho\left(\beta^{2}+2 \beta\right)$. Then, for all $t \in\left[0, t_{1}\right]$, it holds that for some $\hat{r}>r_{0}$ and $\hat{R}<R_{0}$

$$
\Omega_{t} \in S_{\hat{r}, \hat{R}}
$$

where

$$
t_{1}=t_{1}\left(r, R,\left\|\gamma_{\delta}\left(\left|\Xi_{t}\right|\right)\right\|_{L^{\infty}([0, T])}\right):=\frac{1}{2}\left(\min \left\{\frac{\sqrt{r^{2}+\rho^{2}}-\sqrt{r_{0}^{2}+\rho^{2}}}{\mathcal{M}_{1}}, \frac{R_{0}-R}{\mathcal{M}_{1}}\right\}\right)^{2}
$$

Here, $\mathcal{M}_{1}$ is given in Lemma B.1.

Proof. From Lemma B.1, it holds that in $\left[0, t_{1}\right]$

$$
\bar{B}_{(1+\beta) \rho-\mathcal{M}_{1} t_{1}^{\frac{1}{2}}} \subset \Omega_{t} \subset B_{R+\mathcal{M}_{1} t_{1}^{\frac{1}{2}}}
$$

Theorem B.2 implies that $\Omega_{t}$ satisfies $\rho$-reflection for all $t \in\left[0, t_{1}\right]$

By (B.3), it holds that

$$
R+\mathcal{M}_{1} t_{1}^{\frac{1}{2}}<R_{0} \text { and } r_{0}>\left(\left((1+\beta) \rho-\mathcal{M}_{1} t_{1}^{\frac{1}{2}}\right)^{2}-\rho^{2}\right)^{\frac{1}{2}}
$$

From (C.9), we conclude.

Recall definition of energy solutions and comparison principle from [KK18. 
Definition B.4. [KK18, Definition 5.2] Let $\left(\Xi_{t}\right)_{t \geq 0}$ be a energy solution if there exists a sequence $h_{k} \rightarrow 0$ such that

Here, $E_{t}=E_{t}(h)$ is given in (3.10).

$$
d_{H}\left(\Xi_{t}, E_{t}\left(h_{k}\right)\right) \rightarrow 0
$$

Lemma B.5. [KK18, Proposition 6.1] Suppose that $\Omega_{t} \in S_{r, R}$ in $[0, T]$ for some $r>r_{0}$ and $R<R_{0}$. If $\Omega_{0} \subset \subset \Xi_{0}$, then $\Omega_{t} \subset \subset \Xi_{t}$ in $[0, T]$. Also, if $\Xi_{0} \subset \subset \Omega_{0}$, then $\Xi_{t} \subset \subset \Omega_{t}$ in $[0, T]$.

\section{Proof of Proposition 3.4}

Let $\Omega_{t}{ }^{\varepsilon,+}$ and $\Omega_{t}{ }^{\varepsilon,-}$ be viscosity solutions of $V=-H+\gamma_{\delta}\left(\left|\Xi_{t}\right|\right)$ starting from $\Omega_{0}{ }^{\varepsilon,-}:=(1-\varepsilon) \Omega_{0}$ and $\Omega_{0}^{\varepsilon,+}:=(1+\varepsilon) \Omega_{0}$, respectively. Note that

$$
\bar{B}_{\left(1+\beta_{1}\right) \rho} \subset \Omega_{0} \in S_{r_{1}, R_{1}}
$$

where $r_{1}\left(=\rho\left(\beta_{1}^{2}+2 \beta_{1}\right)^{\frac{1}{2}}\right)$ and $\beta_{1}$ are given in from (3.6) and $R_{1}$ from Proposition 3.2. From (B.7), there exist $\varepsilon_{0}>0, \beta \in\left(0, \beta_{1}\right)$ and $R \in\left(R_{1}, R_{0}\right)$ such that

$$
\bar{B}_{(1+\beta) \rho} \subset \Omega_{0}{ }^{\varepsilon, \pm} \in S_{r, R} \text { for all } \varepsilon \in\left[0, \varepsilon_{0}\right] \text {, and } r:=\rho\left(\beta^{2}+2 \beta\right)^{\frac{1}{2}}>r_{0}
$$

Here, $r_{0}$ and $R_{0}$ is given in (3.9).

Let us show that $\Xi_{t}=\Omega_{t}$ in $\left[0, t_{1}\right]$ for $t_{1}=t_{1}\left(r, R, K_{1}\right)$ given in (B.3) and $K_{1}:=\frac{1}{\delta} \max \left\{1,\left|B_{R_{0}}\right|\right\}$. As $\left|\gamma_{\delta}\left(\left|\Xi_{t}\right|\right)\right| \leq K_{1}$, we can apply Lemma B.3 combining with (B.8) and conclude that for some $\hat{r}>r_{0}$ and $\hat{R}<R_{0}$

$$
\Omega_{t}^{\varepsilon, \pm} \in S_{\hat{r}, \hat{R}} \text { in }\left[0, t_{1}\right]
$$

By Lemma B.5, we conclude that

$$
\Omega_{t}^{\varepsilon,-} \subset \Xi_{t} \subset \Omega_{t}^{\varepsilon,+} .
$$

Note that $\Omega_{t}^{\varepsilon,+}$ and $\Omega_{t}{ }^{\varepsilon,-}$ converges to $\Omega_{t}$ as $\varepsilon \rightarrow 0$ from the uniqueness in KK18, Theorem 4.3]. We conclude that $\Xi_{t}=\Omega_{t}$ in $\left[0, t_{1}\right]$. As the proof of Theorem 6.5 in [KK18, we can iterate this step to conclude.

\section{Appendix C. Geometric Properties}

In this section, we consider geometric properties of $\rho$-reflection and $S_{r}$. First, let us recall a local property of $S_{r}$ from [KK18.

Lemma C.1. [KK18, Lemma 3.2] For a continuously differentiable and bounded function $\phi: \mathbb{R}^{n} \rightarrow \mathbb{R}$, let us denote the positive set of $\phi$ by $\Omega(\phi)$. Let us assume that $\Omega(\phi)$ contains $B_{r}(0)$ and $D \phi \neq 0$ on $\partial \Omega(\phi)$. Then the set $\Omega(\phi)$ is in $S_{r}$ if and only if

$$
x \cdot \vec{n}_{x}=x \cdot\left(-\frac{D \phi}{|D \phi|}(x)\right) \geq r \text { for all } x \in \partial \Omega(\phi),
$$

where $\vec{n}_{x}$ denotes the outward normal of $\partial \Omega(\phi)$ at $x$.

Here are several properties of $\rho$-reflection and $S_{r}$ from [FK14].

Lemma C.2. [FK14] Suppose that $\Omega$ satisfies $\rho$-reflection. Then, we have

$$
\sup _{x \in \partial \Omega}|x|-\inf _{x \in \partial \Omega}|x| \leq 4 \rho .
$$

Lemma C.3. [FK14, Lemma 3, 9, 10] For a bounded domain $\Omega$ containing $B_{r}(0)$, the following are equivalent:

(i) $\Omega \in S_{r}$.

(ii) For all $x \in \partial \Omega$, there is an interior cone to $\Omega$ :

$$
I C(x, r):=\left(\left(x+C\left(-x, \theta_{x}\right)\right) \cap C\left(x, \frac{\pi}{2}-\theta_{x}\right)\right) \cup B_{r}(0) \subset \Omega
$$

where

$$
\theta_{x}:=\arcsin \frac{r}{|x|} \in\left[0, \frac{\pi}{2}\right] \text { and } C(x, \theta):=\{y|\langle x, y\rangle \geq \cos \theta| x|| y \mid\} .
$$

(iii) There exists $\epsilon>0$ such that for all $x \in \partial \Omega$, there is an exterior cone to $\Omega$ :

$$
E C(x, r):=\left(x+C\left(x, \theta_{x}\right)\right) \cap B_{\epsilon}(x) \subset \Omega^{c} \text { where } \theta_{x}=\arcsin \frac{r}{|x|} .
$$


Lemma 4.3 can be shown by Lemma C.3

\section{Proof of Lemma 4.3}

From Lemma C.3, it holds that for all $x \in \partial E$,

$$
I C(x, r) \subset E \text { and } E C(x, r) \subset E^{c}
$$

where $I C$ is an interior cone given in (C.1), and $E C$ is an exterior cone given in (C.2). Note that as $|x| \leq R$, the angle of both the interior cone and exterior cone, $\theta_{x}$, is bounded from below as follows,

$$
\theta_{x}:=\arcsin \frac{r}{|x|} \geq \arcsin \frac{r}{R}
$$

Thus, for $\eta_{1}(r, R):=\left|I C\left(R e_{1}, r\right) \cap B_{\varepsilon}\left(R e_{1}\right)\right|$, it holds that for $\varepsilon \in(0, r)$

$$
\eta_{1} \varepsilon^{n} \leq\left|I C(x, r) \cap B_{\varepsilon}(x)\right| \leq\left|E \cap B_{\varepsilon}(x)\right|
$$

Here, $e_{1}$ is a unit vector in the positive $x_{1}$ direction. Similarly, it holds that

$$
\left|B_{\varepsilon}(x) \backslash E\right| \geq\left|B_{\varepsilon}(x) \cap E C(x, r)\right| \geq \eta_{1} \varepsilon^{n} .
$$

Here, $w_{n}$ is a volume of a unit ball in $\mathbb{R}^{n}$.

As $E \in S_{r, R}$, there exists $\varepsilon_{0}=\varepsilon(r, R)<r$ such that for all $\varepsilon \in\left(0, \varepsilon_{0}\right)$

$$
B_{\varepsilon}(x) \cap \partial E=(U, f(U))
$$

up to rotation for some Lipschitz function $f=f_{x, \varepsilon}: U \subset B_{\varepsilon}^{n-1}(x) \rightarrow \mathbb{R}$. Note that as $E \in S_{r, R}$, the Lipschitz constant of $f$ is uniformly bounded by some constant $\mathcal{L}=\mathcal{L}(r, R)$.

From Theorem 9.1 in $M a g 12$,

$$
\mathcal{H}^{n-1}\left(B_{\varepsilon}(x) \cap \partial E\right)=\int_{U} \sqrt{1+|\nabla f|^{2}} d x \leq|U| \sqrt{1+\mathcal{L}^{2}} \leq n w_{n} \varepsilon^{n-1} \sqrt{1+\mathcal{L}^{2}}
$$

Thus, (4.3) holds with $\eta_{2}(r, R):=n w_{n} \sqrt{1+\mathcal{L}^{2}}$. On the other hand, from the isoperimetric inequality in [Mag12, Proposition 12.37] and (4.2), we get the lower bound of (4.3).

Lemma C.4. FK14, Lemma 10, 24]

(1) Suppose that $\Omega$ satisfies $\rho$-reflection. Moreover, $\Omega \in S_{r}$ with

$$
r=\left(\inf _{x \in \partial \Omega}|x|^{2}-\rho^{2}\right)^{1 / 2} .
$$

(2) Suppose that $\Omega$ is in $S_{r, R}$. If there exists $\rho>0$ such that $\overline{B_{\rho}(0)} \subset \Omega$ and $\rho^{2} \geq 5\left(R^{2}-r^{2}\right)$, then $\Omega$ satisfies $\rho$-reflection.

For $E, F \subset \mathbb{R}^{n}$, define the Hausdorff distance by

$$
d_{H}(E, F):=\max \left\{\sup _{x \in E} d(x, F), \sup _{x \in F} d(x, E)\right\}
$$

Lemma C.5. FK14, Lemma 23] Consider sets $\Omega_{1}$ and $\Omega_{2}$ in $S_{r, R}$ for $R>r>0$. Then the following holds:

$$
d_{H}\left(\partial \Omega_{1}, \partial \Omega_{2}\right) \lesssim_{r, R} d_{H}\left(\Omega_{1}, \Omega_{2}\right), \quad\left|\Omega_{1} \Delta \Omega_{2}\right| \lesssim_{r, R} d_{H}\left(\Omega_{1}, \Omega_{2}\right),
$$

Lastly, let us show the following property of characteristic functions.

Lemma C.6. Let $\left\{\left(\Omega_{t}^{k}\right)_{t \geq 0}\right\}_{k \in \mathbb{N}}$ be a sequence of sets in $S_{r, R}$ for $0<r<R$. Suppose that $\Omega_{t}^{k}$ converges locally uniformly to $\Omega_{t}^{\infty}$. For a sequence of functions $\left\{u_{k}\right\}_{k \in \mathbb{N} \cup\{+\infty\}}$ defined by

$$
u_{k}:=\chi_{\Omega_{t}^{k}}-\chi_{\left(\Omega_{t}^{k}\right)^{C}} \text { for } k \in \mathbb{N} \cup\{+\infty\}
$$

it holds that

$$
u_{\infty}^{*}=\limsup _{k \rightarrow \infty} \operatorname{su}_{k} \text { and }\left(u_{\infty}\right)_{*}=\liminf _{k \rightarrow \infty} u_{k}
$$

Here, lim sup ${ }^{*}$ and $\lim \inf _{*}$ are given in (2.4). 
Proof. Let us show the first equation in (C.12) only. The second one can be shown by the parallel arguments.

By uniform convergence in finite interval, for any $j \in \mathbb{N}$, there exists $k_{1}>0$ such that for all $k>k_{1}$

$$
d_{H}\left(\Omega_{t}^{k}, \Omega_{t}^{\infty}\right)<\frac{1}{j} .
$$

Thus, for any $x \in \Omega_{t}^{\infty}$ and $k>k_{1}$, there exists $y \in \Omega_{t}^{k}$ such that $|x-y|<\frac{1}{j}$. Thus, we conclude that

$$
\limsup _{k \rightarrow \infty}{ }^{*} u_{k}(x, t)=\lim _{j \rightarrow \infty} \sup \left\{u_{k}(y, s): k \geq j, \quad|y-x| \leq \frac{1}{j}, \quad|s-t| \leq \frac{1}{j}\right\}=1
$$

and $u_{\infty}^{*}(x)=\lim \sup ^{*} u_{k}(x)$ for $x \in \Omega_{t}^{\infty}$.

Note that we have for any sets $\Omega_{1}, \Omega_{2} \in S_{r, R}$

$$
d_{H}\left(\Omega_{1}^{C}, \Omega_{2}^{C}\right) \leq d_{H}\left(\partial \Omega_{1}, \partial \Omega_{2}\right)
$$

Combining this with Lemma C.5. we conclude that $\left(\Omega_{t}^{k}\right)^{C}$ converges locally uniformly to $\left(\Omega_{t}^{\infty}\right)^{C}$. By parallel arguments, for any $x \in\left(\Omega_{t}^{\infty}\right)^{C}$, we conclude that $\limsup _{k \rightarrow \infty}^{*} u_{k}(x, t)=-1$. As $\lim _{k \rightarrow \infty} \sup ^{*} u_{k}$ is upper semicontinuous, we conclude (C.12).

\section{REFERENCES}

[AKS10] Dimitra C Antonopoulou, Georgia Karali, and Israel Michael Sigal. Stability of spheres under volume-preserving mean curvature flow. Dynamics of PDE, 7(4):327-344, 2010.

[And01] Ben Andrews. Volume-preserving anisotropic mean curvature flow. Indiana University mathematics journal, 50(2):783-827, 2001.

[Ath97] Maria Athanassenas. Volume-preserving mean curvature flow of rotationally symmetric surfaces. Commentarii Mathematici Helvetici, 72(1):52-66, 1997.

[Bar13] Guy Barles. An introduction to the theory of viscosity solutions for first-order Hamilton-Jacobi equations and applications. In Hamilton-Jacobi equations: approximations, numerical analysis and applications, pages 49-109. Springer, Heidelberg, Berlin, Heidelberg, 2013.

[BCCN09] Giovanni Bellettini, Vicent Caselles, Antonin Chambolle, and Matteo Novaga. The volume preserving crystalline mean curvature flow of convex sets in. Journal de Mathématiques Pures et Appliquées, 92(5):499-527, 2009.

[Bou08a] Mariane Bourgoing. Viscosity solutions of fully nonlinear second order parabolic equations with $l^{1}$ dependence in time and neumann boundary conditions. Discrete \& Continuous Dynamical Systems-A, 21(3):763-800, 2008.

[Bou08b] Mariane Bourgoing. Viscosity solutions of fully nonlinear second order parabolic equations with $l^{1}$ dependence in time and neumann boundary conditions. existence and applications to the level-set approach. Discrete $\mathcal{E}^{3}$ Continuous Dynamical Systems-A, 21(4):1047-1069, 2008.

[BSS93] Guy Barles, H Mete Soner, and Panagiotis E Souganidis. Front propagation and phase field theory. SIAM Journal on Control and Optimization, 31(2):439-469, 1993.

[CGG91] Yun Gang Chen, Yoshikazu Giga, and Shun'ichi Goto. Uniqueness and existence of viscosity solutions of generalized mean curvature flow equations. Journal of differential geometry, 33(3):749-786, 1991.

[CSS05] Luis A Caffarelli, Sandro Salsa, and S Salsa. A geometric approach to free boundary problems, volume 68. American Mathematical Soc., 2005.

[Eck04] Klaus Ecker. Regularity theory for mean curvature flow. Springer Science \& Business Media, Boston, MA, 2004.

[EG92] Lawrence C Evans and Ronald F Gariepy. Measure theory and fine properties of functions. Studies in Advanced Mathematics. CRC Press, Boca Raton, FL, 1992.

[EH89] Klaus Ecker and Gerhard Huisken. Mean curvature evolution of entire graphs. Annals of Mathematics, 130(3):453-471, 1989.

[EH91] Klaus Ecker and Gerhard Huisken. Interior estimates for hypersurfaces moving by mean curvature. Inventiones mathematicae, 105(3):547-569, 1991.

[ES98] Joachim Escher and Gieri Simonett. The volume preserving mean curvature flow near spheres. Proceedings of the American Mathematical Society, 126(9):2789-2796, 1998.

[FK14] William M Feldman and Inwon C Kim. Dynamic stability of equilibrium capillary drops. Archive for Rational Mechanics and Analysis, 211(3):819-878, 2014.

[Gig06] Yoshikazu Giga. Surface Evolution Equations, volume 99 of Monographs in Mathematics. Birkhäuser Basel, Basel, 2006.

[GT15] David Gilbarg and Neil S Trudinger. Elliptic partial differential equations of second order. springer, 2015.

[Hui87] Gerhard Huisken. The volume preserving mean curvature flow. J. reine angew. Math, 382(35-48):78, 1987.

[Ish85] Hitoshi Ishii. Hamilton-Jacobi equations with discontinuous Hamiltonians on arbitrary open sets. 28:33-77, 1985.

[KK18] Inwon Kim and Dohyun Kwon. On mean curvature flow with forcing. arXiv:1512.07549 [math.AP], 2018. http://arxiv.org/abs/1512.07549

[Li09] Haozhao Li. The volume-preserving mean curvature flow in Euclidean space. Pacific Journal of Mathematics, 243(2):331-355, December 2009. 
[Mag12] Francesco Maggi. Sets of finite perimeter and geometric variational problems. An Introduction to Geometric Measure Theory. Cambridge University Press, 2012.

[MSS16] Luca Mugnai, Christian Seis, and Emanuele Spadaro. Global solutions to the volume-preserving mean-curvature flow. Calculus of Variations and Partial Differential Equations, 55(1):Art. 18-23, 2016.

[Tak17] Keisuke Takasao. Existence of weak solution for volume preserving mean curvature flow via phase field method. Indiana Univ. Math. J., 66:2015-2035, 2017.

Department of Mathematics, UCla, Los Angeles, USA

E-mail address: ikim@math.ucla.edu

Department of Mathematics, UCla, Los Angeles, USA

E-mail address: dhkwon@ucla.edu 